\title{
NONABELIAN COHOMOLOGY WITH COEFFICIENTS IN LIE GROUPS
}

\author{
JINPENG AN AND ZHENGDONG WANG
}

\begin{abstract}
In this paper we prove some properties of the nonabelian cohomology $H^{1}(A, G)$ of a group $A$ with coefficients in a connected Lie group $G$. When $A$ is finite, we show that for every $A$-submodule $K$ of $G$ which is a maximal compact subgroup of $G$, the canonical map $H^{1}(A, K) \rightarrow H^{1}(A, G)$ is bijective. In this case we also show that $H^{1}(A, G)$ is always finite. When $A=\mathbb{Z}$ and $G$ is compact, we show that for every maximal torus $T$ of the identity component $G_{0}^{\mathbb{Z}}$ of the group of invariants $G^{\mathbb{Z}}, H^{1}(\mathbb{Z}, T) \rightarrow H^{1}(\mathbb{Z}, G)$ is surjective if and only if the $\mathbb{Z}$-action on $G$ is 1 -semisimple, which is also equivalent to the fact that all fibers of $H^{1}(\mathbb{Z}, T) \rightarrow H^{1}(\mathbb{Z}, G)$ are finite. When $A=\mathbb{Z} / n \mathbb{Z}$, we show that $H^{1}(\mathbb{Z} / n \mathbb{Z}, T) \rightarrow H^{1}(\mathbb{Z} / n \mathbb{Z}, G)$ is always surjective, where $T$ is a maximal compact torus of the identity component $G_{0}^{\mathbb{Z} / n \mathbb{Z}}$ of $G^{\mathbb{Z} / n \mathbb{Z}}$. When $A$ is cyclic, we also interpret some properties of $H^{1}(A, G)$ in terms of twisted conjugate actions of $G$.
\end{abstract}

\section{INTRODUCTION}

Let $A$ be a group, and let $G$ be a (nonabelian) $A$-module. A map $\alpha: A \rightarrow G$ $\left(a \mapsto g_{a}\right)$ is called a cocycle if $g_{a b}=g_{a} a\left(g_{b}\right)$. The set of all cocycles is denoted by $Z^{1}(A, G)$. Two cocycles $\alpha_{1}, \alpha_{2}$ are cohomologous if there exists $g \in G$ such that $\alpha_{2}(a)=g^{-1} \alpha_{1}(a) a(g)$ for every $a \in A$. This is an equivalence relation in $Z^{1}(A, G)$. The nonabelian cohomology $H^{1}(A, G)$ of $A$ with coefficients in $G$ is defined to be the set of all equivalence classes in $Z^{1}(A, G)$.

Most studies of this kind of cohomology concentrate on the case that $G$ is an algebraic group, which plays an important role in the study of algebraic groups and number theory (see, for example, [9] and [10]). In this paper we consider the case that $G$ is a connected Lie group, and prove some properties of $H^{1}(A, G)$. Some of these properties provide a way to compute $H^{1}(A, G)$. When $A$ is a cyclic group, we also relate $H^{1}(A, G)$ with the so-called twisted conjugate actions of $G$, and interpret some properties of $H^{1}(A, G)$ as properties concerning structures of orbits of twisted conjugate actions.

Throughout this paper, we make the convention that when we say $G$ is a Lie group with a nonabelian $A$-module structure, we always assume that $A$ acts on $G$ by automorphisms of Lie groups, that is, $A$ acts smoothly on $G$; and when we say

Received by the editors September 17, 2005 and, in revised form, March 14, 2006.

2000 Mathematics Subject Classification. Primary 20J06, 22E15, 57S15, 57S20.

Key words and phrases. Nonabelian cohomology, Lie group, twisted conjugate action.

This work was supported by the 973 Project Foundation of China (\#TG1999075102). 
that $f: H \rightarrow G$ is an $A$-homomorphism of such two $A$-modules, we always assume that $f$ is also a homomorphism of Lie groups, that is, $f$ is smooth.

Two cases regarding the type of the group $A$ will be considered; that is, $A$ is a finite group and $A$ is the infinite cyclic group $\mathbb{Z}$. For the first case, if $A$ is a finite cyclic group $\mathbb{Z} / n \mathbb{Z}$, further properties of $H^{1}(\mathbb{Z} / n \mathbb{Z}, G)$ will be proved.

For the case that $A$ is finite, we have

Theorem 1.1. Let $A$ be a finite group, and let $G$ be a connected Lie group with an A-module structure. Then

(i) There exists a maximal compact subgroup $K$ of $G$ which is an A-submodule of $G$;

(ii) For each maximal compact subgroup $K$ of $G$ which is also an A-submodule of $G$, the canonical map $H^{1}(A, K) \rightarrow H^{1}(A, G)$ is bijective.

Theorem 1.1 reduces the calculation of $H^{1}(A, G)$ to the calculation of the cohomology of $A$ with coefficients in a compact Lie group. In the case that $G$ is the complexification of a compact Lie group $K$ and $\mathbb{Z} / 2 \mathbb{Z}$ acts on $G$ by complex conjugation, the bijectivity of the map $H^{1}(\mathbb{Z} / 2 \mathbb{Z}, K) \rightarrow H^{1}(\mathbb{Z} / 2 \mathbb{Z}, G)$ has been proved in Serre [13], Chapter III, Section 4.5.

Let $\operatorname{Map}(A, G)$ denote the set of all maps from $A$ to $G$. If $A$ is finite, there is a natural identification $\operatorname{Map}(A, G) \cong G^{|A|}$, where $|A|$ is the order of $A$. Hence $\operatorname{Map}(A, G)$ inherits a structure of smooth manifold from $G^{|A|}$. The set of cocycles $Z^{1}(A, G)$ is a closed subset of $\operatorname{Map}(A, G)$. The following assertion will be proved.

Theorem 1.2. Let $A$ be a finite group, and let $G$ be a connected Lie group with an $A$-module structure. Then we have

(i) $Z^{1}(A, G) \subset \operatorname{Map}(A, G)$ has finitely many connected components, each of which is a closed submanifold of $\operatorname{Map}(A, G)$;

(ii) Each cohomology class in $Z^{1}(A, G)$ is a connected component of $Z^{1}(A, G)$.

Theorem 1.2 has the following obvious corollary.

Corollary 1.1. Let $A$ be a finite group, and let $G$ be a connected Lie group with an $A$-module structure. Then $H^{1}(A, G)$ is finite.

Corollary 1.1 generalizes the finiteness theorem for Galois cohomology with coefficients in algebraic groups defined over $\mathbb{R}$ (see [13], Chapter III, Section 4.3).

For the case that $A=\mathbb{Z}$, we restrict our attention to the case that $G$ is compact. To state our theorem, we first introduce a notion.

An automorphism $\sigma$ of a connected compact Lie group $G$ is 1-semisimple if $\operatorname{ker}(1-d \sigma)=\operatorname{ker}\left((1-d \sigma)^{2}\right)$ in the Lie algebra $\mathfrak{g}$ of $G$. All automorphisms of a compact semisimple Lie group are 1-semisimple, and then $\sigma$ is 1-semisimple if and only if the restriction of $\sigma$ to the connected component of the center of $G$ is 1 -semisimple. For a connected compact Lie group $G$ with a $\mathbb{Z}$-module structure, the $\mathbb{Z}$-action on $G$ is 1 -semisimple if a generator of $\mathbb{Z}$ acts 1 -semisimply on $G$.

For a Lie group $G$ with an $A$-module structure, we always denote the group of invariants by $G^{A}$, and denote the identity component of $G^{A}$ by $G_{0}^{A}$. The following theorem will be proved.

Theorem 1.3. Let $G$ be a connected compact Lie group with a $\mathbb{Z}$-module structure, and let $T$ be a maximal torus of $G_{0}^{\mathbb{Z}}$. Let $i: T \rightarrow G$ be the canonical inclusion, and 
let $i_{1}: H^{1}(\mathbb{Z}, T) \rightarrow H^{1}(\mathbb{Z}, G)$ be the canonical map induced by $i$. Then the following statements are equivalent:

(i) The $\mathbb{Z}$-action on $G$ is 1-semisimple;

(ii) The map $i_{1}$ is surjective;

(iii) The kernel of $i_{1}$ is finite;

(iv) All fibers of $i_{1}$ are finite.

We note here that when we say that a set is finite, we allow it to be empty. We will use the word "nonempty and finite" if we want to emphasize that it is nonempty.

If $A$ is a finite cyclic group $\mathbb{Z} / n \mathbb{Z}$, we have further properties about $H^{1}(A, G)$ besides Theorems 1.1 and 1.2 .

For a Lie group $H$, a subgroup $T$ of $H$ is a maximal compact torus of $H$ if $T$ is a compact torus and there is no other compact torus $T^{\prime}$ of $H$ such that $T \varsubsetneqq T^{\prime}$. In fact, any two maximal compact tori are conjugate (see Section 5). The following assertion will be proved.

Theorem 1.4. Let $G$ be a connected Lie group with a $\mathbb{Z} / n \mathbb{Z}$-module structure, and let $T$ be a maximal compact torus of $G_{0}^{\mathbb{Z} / n \mathbb{Z}}$. Then the canonical map $i_{1}$ : $H^{1}(\mathbb{Z} / n \mathbb{Z}, T) \rightarrow H^{1}(\mathbb{Z} / n \mathbb{Z}, G)$ is surjective.

A theorem of T. A. Springer (see Serre [13], Chapter III, Section 4.3, Lemma 6) says that if $G$ is a linear algebraic group defined over a perfect field $k$ with a Cartan subgroup $C$, then the canonical map $H^{1}\left(k, N_{G}(C)\right) \rightarrow H^{1}(k, G)$ between sets of Galois cohomology is surjective. Theorem 1.4 may be viewed as a generalization of the theorem of Springer in the case that $k=\mathbb{R}$.

The set of cocycles $Z^{1}(\mathbb{Z} / n \mathbb{Z}, G)$ may be identified with a closed subset $Z=$ $\left\{g \in G \mid g \sigma(g) \cdots \sigma^{n-1}(g)=e\right\}$ of $G$, where $\sigma$ is a generator of $\mathbb{Z} / n \mathbb{Z}$ (see Section 2 ). Under this identification, Theorem 1.2 has the following variant form.

Theorem 1.5. Let $G$ be a connected Lie group with a $\mathbb{Z} / n \mathbb{Z}$-module structure, and let $\sigma$ be a generator of $\mathbb{Z} / n \mathbb{Z}$. Let $Z=\left\{g \in G \mid g \sigma(g) \cdots \sigma^{n-1}(g)=e\right\}$. Then we have

(i) $Z$ has finitely many connected components, each of which is a closed submanifold of $G$;

(ii) Under the identification $Z^{1}(\mathbb{Z} / n \mathbb{Z}, G) \cong Z$, each cohomology class is a connected component of $Z$.

When $A$ is cyclic, that is, $A=\mathbb{Z}$ or $\mathbb{Z} / n \mathbb{Z}$, the first nonabelian cohomology $H^{1}(A, G)$ has a closed relation with the so-called twisted conjugate actions of $G$ on $G$. For a connected Lie group $G$ with an automorphism $\sigma$, the twisted conjugate action $\tau: G \times G \rightarrow G$ of $G$ on $G$ associated with $\sigma$ is defined by $\tau_{g}(h)=g h \sigma(g)^{-1}$. An orbit of the twisted conjugate action is called a twisted orbit. This kind of action was introduced in $[1,2]$ where an equivariant embedding of a symmetric space $G / K$ into $G$ (or a covering group of $G$ ) was established, and the image of the embedding is just the twisted orbit through the identity element of $G$. This embedding was then applied to the theory of random matrix ensembles associated with symmetric spaces in $[4,5]$, transforming the integration manifold of a random matrix ensemble from a symmetric space modelled by a matrix group to a space of matrices. 
The relation between twisted conjugate actions and nonabelian cohomology may be described briefly as follows. An automorphism $\sigma$ of a Lie group $G$ naturally induces a $\mathbb{Z}$-module structure on $G$ by $(m, g) \mapsto \sigma^{m}(g)$, where $m \in \mathbb{Z}, g \in G$. The set of cocycles $Z^{1}(\mathbb{Z}, G)$ may be identified naturally with $G$, and two cocycles $z_{1}, z_{2} \in G$ are cohomologous if and only if they lie in the same twisted orbit. So $H^{1}(\mathbb{Z}, G)$ may be identified with the space of twisted orbits. If moreover $\sigma$ has finite order dividing a positive integer $n$, the associated $\mathbb{Z}$-module structure on $G$ reduces to a $\mathbb{Z} / n \mathbb{Z}$-module structure on $G$. The set of cocycles $Z^{1}(\mathbb{Z} / n \mathbb{Z}, G)$ may be identified naturally with $Z=\left\{g \in G \mid g \sigma(g) \cdots \sigma^{n-1}(g)=e\right\}$, which is invariant under the twisted conjugate action. Two cocycles $z_{1}, z_{2} \in Z$ are cohomologous if and only if they lie in the same twisted orbit. Hence $H^{1}(\mathbb{Z} / n \mathbb{Z}, G)$ may be identified with the set of twisted orbits which are contained in $Z$. More details about this relation will be given in Section 2 .

With this relation in hand, we will interpret some properties of $H^{1}(A, G)$ mentioned above in the language of twisted conjugate actions. Some of these interpretations are as follows.

Theorem 1.6. Let $G$ be a connected compact Lie group, and let $\sigma$ be an automorphism of $G$. Let $T$ be a maximal torus of $G_{0}^{\sigma}$. Then the following statements are equivalent:

(i) $\sigma$ is 1-semisimple;

(ii) $O \cap T$ is nonempty for every twisted orbit $O$ of $G$ associated with $\sigma$;

(iii) $O_{e} \cap T$ is finite, where $O_{e}$ is the twisted orbit through the identity element $e$;

(iv) $O \cap T$ is finite for every twisted orbit $O$ of $G$ associated with $\sigma$.

Theorem 1.7. Let $G$ be a connected Lie group, and let $\sigma$ be an automorphism of $G$ of finite order. Let $Z=\left\{g \in G \mid g \sigma(g) \cdots \sigma^{n-1}(g)=e\right\}$, where $n$ is a positive integer which is divisible by the order of $\sigma$. Then

(i) $Z$ has finitely many connected components, each of which is a twisted orbit;

(ii) For every maximal compact torus $T$ of $G_{0}^{\sigma}$ and every twisted orbit $O$ contained in $Z, O \cap T$ is nonempty and finite;

(iii) There exists a maximal compact subgroup $K$ of $G$ which is $\sigma$-invariant. For every such $K$ and every twisted orbit $O$ of $G$ contained in $Z, O \cap K$ is a twisted orbit of $K$.

Now we give a sketch of the contents of each of the following sections. In Section 2 we will review some basic facts on nonabelian cohomology. The relation between nonabelian cohomology of cyclic groups and twisted conjugate actions will also be given.

Section 3 will concern properties of nonabelian cohomology of finite groups with coefficients in Lie groups. Theorems 1.1 and 1.2 will be proved. A key ingredient in the proof of Theorem 1.1 is the conjugacy theorem for maximal compact subgroups of Lie groups with finitely many connected components. The proof of Theorem 1.2 makes use of a theorem of Weil for smooth manifolds, which we call Weil's Lemma (Fact 3.2).

In Section 4 we will prove Theorem 1.3. Note that Theorem 1.3 is equivalent to Theorem 1.6, which says, among other things, that if the automorphism $\sigma$ is 1-semisimple, then every twisted orbit intersects every maximal torus of $G_{0}^{\sigma}$. This fact is a generalization of Cartan's conjugacy theorem for compact Lie groups, 
which is the special case that $\sigma$ is the identity in Theorem 1.6. Besides using cohomology exact sequences and twisting processes, an important ingredient in the proof of Theorem 1.3 is the Lefschetz Fixed Point Theorem. This is reflected in the proof of Lemma 4.1. Our proof of Lemma 4.1 follows the idea of Weil's topological proof of Cartan's conjugacy theorem [15]. We also show that under the condition of 1-semisimplicity, each connected component of $H^{0}(\mathbb{Z}, G / T)$ is a closed homogeneous submanifold of $G / T$, and the restriction of the coboundary operator $\delta: H^{0}(\mathbb{Z}, G / T) \rightarrow H^{1}(\mathbb{Z}, T)$ to each connected component of $H^{0}(\mathbb{Z}, G / T)$ is constant.

Theorems 1.4 and 1.5 will be proved in Section 5. The proof of Theorem 1.4 will make use of Theorems 1.1 and 1.3. A corollary of Theorem 1.4 says that if $G$ is simply connected and solvable, then $H^{1}(\mathbb{Z} / n \mathbb{Z}, G)$ is trivial, due to the fact that $G$ admits no nontrivial compact torus. Theorem 1.5 is in fact equivalent to the particular case of Theorem 1.2 when $A$ is finite cyclic. We will provide another proof of Theorem 1.5 in Section 5, without the use of Weil's Lemma. We also have the result that each connected component of $H^{0}(\mathbb{Z} / n \mathbb{Z}, G / T)$ is a closed homogeneous submanifold of $G / T$, and the restriction of the coboundary operator $\delta: H^{0}(\mathbb{Z} / n \mathbb{Z}, G / T) \rightarrow H^{1}(\mathbb{Z} / n \mathbb{Z}, T)$ to each connected component of $H^{0}(\mathbb{Z} / n \mathbb{Z}, G / T)$ is constant.

Section 6 will be devoted to properties of twisted conjugate actions of Lie groups. We will first present some elementary properties of such actions. Then we interpret some results proved in Sections 3-5 in the language of twisted conjugate actions. Theorems 1.6 and 1.7 will be proved. In fact, Theorem 1.6 is equivalent to Theorem 1.3, item (i) of Theorem 1.7 is equivalent to Theorem 1.5, item (ii) of Theorem 1.7 can be implied from Corollary 1.1 and Theorem 1.4, and item (iii) of Theorem 1.7 can be implied from Theorem 1.1.

The authors would like to thank the two referees for suggestions on improving the presentation of the content (especially the comment of considering cohomology of general finite groups) and for suggestions on improving the English usage. The first author is in debt to Professor Jiu-Kang Yu for many valuable conversations which engaged the author in the subject of nonabelian cohomology. He would also like to thank Professors F. Fang, Z. Hajto, and K.-H. Neeb for valuable conversations or kind help.

\section{Preliminaries on NONABElian COHOMOlOGY}

In this section we list some facts of nonabelian cohomology which will be used later, and explain the relation between nonabelian cohomology and twisted conjugate actions. Most of these facts may be found in Serre $[12,13]$.

Let $A$ be a group, and let $X$ be an $A$-set. By definition, the zeroth nonabelian cohomology $H^{0}(A, X)$ of $A$ with coefficients in $X$ is the set $X^{A}=\{x \in X \mid a(x)=$ $x, \forall a \in A\}$. Now let $G$ be a group (which is always a Lie group in subsequent sections) on which $A$ acts by automorphisms, that is, $G$ is a nonabelian $A$-module; then $H^{0}(A, G)=G^{A}$ is a subgroup of $G$. A map $\alpha: A \rightarrow G\left(a \mapsto g_{a}\right)$ is called a cocycle if $g_{a b}=g_{a} a\left(g_{b}\right)$. (This forces that $\alpha$ maps the identity element of $A$ to the identity element of $G$.) The set of all cocycles is denoted by $Z^{1}(A, G)$. Two cocycles $\alpha_{1}, \alpha_{2} \in Z^{1}(A, G)$ are cohomologous if there exists $g \in G$ such that $\alpha_{2}(a)=$ $g^{-1} \alpha_{1}(a) a(g)$ for every $a \in A$. This is an equivalence relation in $Z^{1}(A, G)$, and the first nonabelian cohomology $H^{1}(A, G)$ of $A$ with coefficients in $G$ is defined to be 
the pointed set of all equivalence classes in $Z^{1}(A, G)$ with neutral element the class of the unit cocycle. It is obvious that $H^{1}(A, G \times H) \cong H^{1}(A, G) \times H^{1}(A, H)$. Note that for nonabelian modules, only the zeroth and first cohomology are commonly used. For the definition of higher cohomology, see the references in $[12,13]$.

Here is an equivalent description of $H^{1}(A, G)$. Let $\alpha: A \rightarrow G\left(a \mapsto g_{a}\right)$ be a cocycle. Construct a map $\widetilde{\alpha}: A \rightarrow G \rtimes A$ by $\widetilde{\alpha}(a)=\left(g_{a}, a\right)$. Then $\widetilde{\alpha}$ is homomorphism of groups. Conversely, if $\widetilde{\alpha}: A \rightarrow G \rtimes A$ is a homomorphism splitting the exact sequence $0 \rightarrow G \rightarrow G \rtimes A \rightarrow A \rightarrow 0$, then $p_{1} \circ \widetilde{\alpha}$ is a cocycle, where $p_{1}: G \rtimes A \rightarrow G$ is the projection to the first factor. So $Z^{1}(A, G)$ may be identified with the set of homomorphisms from $A$ to $G \rtimes A$ splitting the exact sequence $0 \rightarrow G \rightarrow G \rtimes A \rightarrow A \rightarrow 0$. Under this identification, it is easy to show that two homomorphisms $\widetilde{\alpha}_{1}, \widetilde{\alpha}_{2}: A \rightarrow G \rtimes A$ are cohomologous if and only if there exists $g \in G$ such that $\widetilde{\alpha}_{2}=g^{-1} \widetilde{\alpha}_{1} g$.

Let $f: H \rightarrow G$ be a homomorphism of nonabelian $A$-modules. Then there are canonical induced maps of pointed sets $f_{i}: H^{i}(A, H) \rightarrow H^{i}(A, G), i=0,1$. $f_{0}$ is also a homomorphism of groups. If $H$ is an $A$-submodule of $G$, then $G / H$ is an $A$-set, and we have an exact sequence of pointed sets

$$
0 \rightarrow H^{0}(A, H) \stackrel{f_{0}}{\rightarrow} H^{0}(A, G) \rightarrow H^{0}(A, G / H) \stackrel{\delta}{\rightarrow} H^{1}(A, H) \stackrel{f_{1}}{\rightarrow} H^{1}(A, G),
$$

where the coboundary operator $\delta$ maps an element $g H \in H^{0}(A, G / H)$ to the class of the cocycle $g_{a}=g^{-1} a(g)$, and the neutral element of $H^{0}(A, G / H)$ is chosen to be $H \in G / H$.

Let $G$ be a nonabelian $A$-module, and let $\alpha \in Z^{1}(A, G)$. One can define another $A$-module $G_{\alpha}$ by twisting $G$ using the cocycle $\alpha$ as follows. The underlying group of $G_{\alpha}$ is $G$, and $A$ acts on $G_{\alpha}$ by $t_{a}(g)=\alpha(a) a(g) \alpha(a)^{-1}$. Then the map $Z^{1}\left(A, G_{\alpha}\right) \rightarrow Z^{1}(A, G)$ defined by $\beta \mapsto \beta \cdot \alpha$ is a bijection, and induces a bijection $t_{\alpha}: H^{1}\left(A, G_{\alpha}\right) \rightarrow H^{1}(A, G)$, under which the neutral element of $H^{1}\left(A, G_{\alpha}\right)$ is mapped to the cohomology class $[\alpha]$ of $\alpha$. If $f: H \rightarrow G$ is an $A$-homomorphism of nonabelian $A$-modules, and if $\alpha \in Z^{1}(A, H)$, then $f_{\alpha}=f: H_{\alpha} \rightarrow G_{f \circ \alpha}$ is also an $A$-homomorphism, and we have the following commutative diagram:

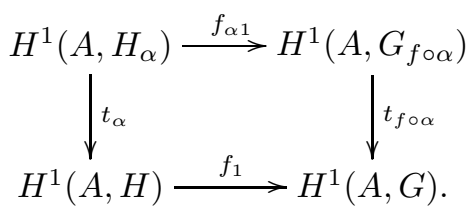

So $t_{\alpha}$ is a bijection between $\operatorname{ker}\left(f_{\alpha 1}\right)$ and the fiber $f_{1}^{-1}\left(f_{1}([\alpha])\right)$. This means that the twisting process allows one to transform each nonempty fiber of $f_{1}$ to a kernel.

Now we describe the relation between nonabelian cohomology and twisted conjugate actions. Let $G$ be a group with an automorphism $\sigma$. The twisted conjugate action $\tau$ of $G$ on itself associated with $\sigma$ is defined by $\tau_{g}(h)=g h \sigma(g)^{-1}$. An orbit of the twisted conjugate action is called a twisted orbit. The automorphism $\sigma$ of $G$ induces in a natural way a $\mathbb{Z}$-action $\mathbb{Z} \times G \rightarrow G$ on $G$, defined by $(m, g) \mapsto \sigma^{m}(g)$. This makes $G$ a nonabelian $\mathbb{Z}$-module. A cocycle in $Z^{1}(\mathbb{Z}, G)$ is determined by its value on $1 \in \mathbb{Z}$. In fact, for a cocycle $\mathbb{Z} \rightarrow G\left(m \mapsto g_{m}\right)$, we have $g_{m}=g_{1} \sigma\left(g_{1}\right) \cdots \sigma^{m-1}\left(g_{1}\right), g_{-m}=\sigma^{-1}\left(g_{1}^{-1}\right) \cdots \sigma^{-m}\left(g_{1}^{-1}\right), m \geq 1$. Conversely, each $g_{1} \in G$ determines a cocycle in this way. So we may identify $Z^{1}(\mathbb{Z}, G)$ with $G$. Under this identification, two cocycles $z_{1}, z_{2} \in G$ are cohomologous if and 
only if there exists $g \in G$ such that $z_{2}=g^{-1} z_{1} \sigma(g)$, that is, $z_{1}$ and $z_{2}$ lie in the same twisted orbit. So $H^{1}(\mathbb{Z}, G)$ may be identified with the space of twisted orbits.

Let $H$ be a subgroup of $G$ which is $\sigma$-invariant. Then $H$ is a $\mathbb{Z}$-submodule of $G$. With the identification $Z^{1}(\mathbb{Z}, G) \cong G$ in mind, it is clear that saying the canonical map $H^{1}(\mathbb{Z}, H) \rightarrow H^{1}(\mathbb{Z}, G)$ is surjective is equivalent to saying that the intersection of every twisted orbit of $G$ with $H$ is nonempty, and saying that $H^{1}(\mathbb{Z}, H) \rightarrow H^{1}(\mathbb{Z}, G)$ is bijective is equivalent to saying that the intersection of every twisted orbit of $G$ with $H$ is just one twisted orbit of $H$. In particular, if $H \subset G^{\sigma}=\{g \in G \mid \sigma(g)=g\}$ and $H$ is abelian, then $H^{1}(\mathbb{Z}, H)=Z^{1}(\mathbb{Z}, H)$, hence saying the kernel of $H^{1}(\mathbb{Z}, H) \rightarrow H^{1}(\mathbb{Z}, G)$ is finite is equivalent to saying that the intersection of the twisted orbit through the identity element $e$ of $G$ with $H$ is a finite set, and saying that all fibers of $H^{1}(\mathbb{Z}, H) \rightarrow H^{1}(\mathbb{Z}, G)$ are finite is equivalent to saying that the intersection of every twisted orbit with $H$ is a finite set.

If $\sigma$ is of finite order, the associated $\mathbb{Z}$-module structure on $G$ reduces to a $\mathbb{Z} / n \mathbb{Z}$ module structure on $G$ for each positive integer $n$ which is divisible by the order of $\sigma$. Let $\mathbb{Z} / n \mathbb{Z} \rightarrow G\left(m \mapsto g_{m}\right)$ be a cocycle in $Z^{1}(\mathbb{Z} / n \mathbb{Z}, G)$. Then we have $g_{m}=$ $g_{1} \sigma\left(g_{1}\right) \cdots \sigma^{m-1}\left(g_{1}\right), 1 \leq m \leq n-1$, and $g_{1} \sigma\left(g_{1}\right) \cdots \sigma^{n-1}\left(g_{1}\right)=e$. Conversely, each $g_{1} \in G$ satisfying $g_{1} \sigma\left(g_{1}\right) \cdots \sigma^{n-1}\left(g_{1}\right)=e$ determines a cocycle in this way. So we may identify $Z^{1}(\mathbb{Z} / n \mathbb{Z}, G)$ with the subset $Z=\left\{g \in G \mid g \sigma(g) \cdots \sigma^{n-1}(g)=e\right\}$ of $G$, which is invariant under the twisted conjugate action. Under this identification, two cocycles $z_{1}, z_{2} \in Z$ are cohomologous if and only if they lie in the same twisted orbit. Hence $H^{1}(\mathbb{Z} / n \mathbb{Z}, G)$ may be identified with the set of twisted orbits which are contained in $Z$.

If $H$ is a closed subgroup of $G$ which is $\sigma$-invariant, then $H$ is a $\mathbb{Z} / n \mathbb{Z}$-submodule of $G$. Similar to the case of $\mathbb{Z}$-modules, saying the canonical map $H^{1}(\mathbb{Z} / n \mathbb{Z}, H) \rightarrow$ $H^{1}(\mathbb{Z} / n \mathbb{Z}, G)$ is surjective is equivalent to saying that the intersection of every twisted orbit contained in $Z$ with $H$ is nonempty, and so on.

\section{NonAbelian COHOMOlogy OF Finite GROUPS WITH COEFFICIENTS IN LIE GROUPS}

In this section we consider nonabelian cohomology of general finite groups with coefficients in connected Lie groups, and prove Theorems 1.1 and 1.2. The first theorem reads as follows.

Theorem 3.1. Let $A$ be a finite group, and let $G$ be a connected Lie group with an A-module structure. Then

(i) There exists a maximal compact subgroup $K$ of $G$ which is an A-submodule of $G$;

(ii) For each $K$ satisfying the conditions in (i), the canonical map $H^{1}(A, K) \rightarrow$ $H^{1}(A, G)$ is bijective.

To prove Theorem 3.1, we need the following fact, which will also be used later in the proofs of other assertions.

Fact 3.1 ([6], Chapter XV, Theorem 3.1). Let $H$ be a Lie group with finitely many connected components, and let $K$ be a maximal compact subgroup of $H$. Let $\mathfrak{h}$ and $\mathfrak{k}$ be the Lie algebras of $H$ and $K$, respectively. Then we have

(i) $K \cap H_{0}=K_{0}$, and $K_{0}$ is a maximal compact subgroup of $H_{0}$;

(ii) For each compact subgroup $K^{\prime}$ of $H$, there exists $h \in H_{0}$ such that $h K^{\prime} h^{-1} \subset$ $K$ 
(iii) There exist linear subspaces $\mathfrak{m}_{1}, \cdots, \mathfrak{m}_{r}$ of $\mathfrak{h}$ with $\mathfrak{h}=\mathfrak{k} \oplus \mathfrak{m}_{1} \oplus \cdots \oplus \mathfrak{m}_{r}$ such that $\operatorname{Ad}(k)\left(\mathfrak{m}_{i}\right)=\mathfrak{m}_{i}, \forall k \in K, i \in\{1, \cdots, r\}$, and such that the map $\varphi: K \times \mathfrak{m}_{1} \times$ $\cdots \times \mathfrak{m}_{r} \rightarrow H$ defined by $\varphi\left(k, X_{1}, \cdots, X_{r}\right)=k e^{X_{1}} \cdots e^{X_{r}}$ is a diffeomorphism.

Proof of Theorem 3.1. We construct the semidirect product $G \rtimes A$, which is a Lie group with finitely many connected components. We identify the identity component of $G \rtimes A$ with $G$. Then $a(g)=(e, a) g(e, a)^{-1}$ for all $a \in A$ and $g \in G$. Since the subgroup $\{e\} \rtimes A$ of $G \rtimes A$ is compact, there is a maximal compact subgroup $K^{\prime}$ of $G \rtimes A$ such that $\{e\} \rtimes A \subset K^{\prime}$. Let $K=K_{0}^{\prime} \subset G$. By Fact $3.1, K$ is a maximal compact subgroup of $G$. Since $a(K)=(e, a) K(e, a)^{-1}=K$ for all $a \in A$, $K$ is an $A$-submodule of $G$. This proves (i).

Now we prove (ii). Let $K$ be a maximal compact subgroup $K$ of $G$ which is also a $\mathbb{Z} / n \mathbb{Z}$-submodule of $G$. Then $K \rtimes A$ is a maximal compact subgroup of $G \rtimes A$. Note that $Z^{1}(A, G)$ may be identified naturally with the set of homomorphisms $A \rightarrow G \rtimes A$ splitting the exact sequence

$$
0 \rightarrow G \rightarrow G \rtimes A \rightarrow A \rightarrow 0
$$

by identifying a cocycle $\alpha: A \rightarrow G$ with $\widetilde{\alpha}: A \rightarrow G \rtimes A(a \mapsto(\alpha(a), a))$, and $\alpha$ is in $Z^{1}(A, K)$ if and only if $\widetilde{\alpha}$ assumes values in $K \rtimes A$ (see Section 2). Hence to prove $H^{1}(A, K) \rightarrow H^{1}(A, G)$ is surjective, it is sufficient to prove that for every homomorphism $\widetilde{\alpha}: A \rightarrow G \rtimes A$ splitting the above exact sequence, there exists $g \in G$ such that $g^{-1} \widetilde{\alpha} g$ assumes values in $K \rtimes A$. But for such an $\widetilde{\alpha}, \widetilde{\alpha}(A)$ is a finite subgroup of $G \rtimes A$. By Fact 3.1, there exists $g \in G$ such that $g^{-1} \widetilde{\alpha}(A) g \subset K \rtimes A$. This proves that $H^{1}(A, K) \rightarrow H^{1}(A, G)$ is surjective.

Let $\mathfrak{g}, \mathfrak{k}$ be the Lie algebras of $G$ and $K$. By Fact 3.1, there exist linear subspaces $\mathfrak{m}_{1}, \cdots, \mathfrak{m}_{r}$ of $\mathfrak{g}$ with $\mathfrak{g}=\mathfrak{k} \oplus \mathfrak{m}_{1} \oplus \cdots \oplus \mathfrak{m}_{r}$ such that $\operatorname{Ad}(k)\left(\mathfrak{m}_{i}\right)=\mathfrak{m}_{i}, \forall k \in$ $K \rtimes A, 1 \leq i \leq r$, and such that the map $\varphi: K \times \mathfrak{m}_{1} \times \cdots \times \mathfrak{m}_{r} \rightarrow G$ defined by $\varphi\left(k, X_{1}, \cdots, X_{r}\right)=k e^{X_{1}} \cdots e^{X_{r}}$ is a diffeomorphism. To prove the injectivity of $H^{1}(A, K) \rightarrow H^{1}(A, G)$, let $\widetilde{\alpha}_{1}, \widetilde{\alpha}_{2}: A \rightarrow K \rtimes A$ be two homomorphisms splitting the above exact sequence. Suppose there exists $g \in G$ such that $\widetilde{\alpha}_{2}=g^{-1} \widetilde{\alpha}_{1} g$. It is sufficient to show that there exists $k \in K$ such that $\widetilde{\alpha}_{2}=k^{-1} \widetilde{\alpha}_{1} k$. For every $a \in A$, rewrite the equality $\widetilde{\alpha}_{2}(a)=g^{-1} \widetilde{\alpha}_{1}(a) g$ as $\widetilde{\alpha}_{2}(a)^{-1} g \widetilde{\alpha}_{2}(a)=\widetilde{\alpha}_{2}(a)^{-1} \widetilde{\alpha}_{1}(a) g$, and let $g=k e^{X_{1}} \cdots e^{X_{r}}, k \in K, X_{i} \in \mathfrak{m}_{i}$. Then we have

$$
\widetilde{\alpha}_{2}(a)^{-1} k \widetilde{\alpha}_{2}(a) e^{\operatorname{Ad}\left(\widetilde{\alpha}_{2}(a)^{-1}\right) X_{1}} \cdots e^{\operatorname{Ad}\left(\widetilde{\alpha}_{2}(a)^{-1}\right) X_{r}}=\widetilde{\alpha}_{2}(a)^{-1} \widetilde{\alpha}_{1}(a) k e^{X_{1}} \cdots e^{X_{r}},
$$

that is,

$$
\begin{aligned}
& \varphi\left(\widetilde{\alpha}_{2}(a)^{-1} k \widetilde{\alpha}_{2}(a), \operatorname{Ad}\left(\widetilde{\alpha}_{2}(a)^{-1}\right) X_{1}, \cdots, \operatorname{Ad}\left(\widetilde{\alpha}_{2}(a)^{-1}\right) X_{r}\right) \\
= & \varphi\left(\widetilde{\alpha}_{2}(a)^{-1} \widetilde{\alpha}_{1}(a) k, X_{1}, \cdots, X_{r}\right) .
\end{aligned}
$$

Since $\varphi$ is a diffeomorphism, we have $\widetilde{\alpha}_{2}(a)^{-1} k \widetilde{\alpha}_{2}(a)=\widetilde{\alpha}_{2}(a)^{-1} \widetilde{\alpha}_{1}(a) k, \forall a \in A$, that is, $\widetilde{\alpha}_{2}=k^{-1} \widetilde{\alpha}_{1} k$. This proves the injectivity, and then finishes the proof of the theorem.

For two sets $X$ and $Y$, denote the set of all maps from $X$ to $Y$ by $\operatorname{Map}(X, Y)$. Let $A$ be a finite group, and let $G$ be a connected Lie group with an $A$-module structure. Then there is a natural identification $\operatorname{Map}(A, G) \cong \prod_{a \in A} G \cong G^{|A|}$, where $|A|$ is the order of $A$. Hence $\operatorname{Map}(A, G)$ inherits a structure of smooth manifold from $G^{|A|}$. The set of cocycles $Z^{1}(A, G)$ is a closed subset of $\operatorname{Map}(A, G)$. 
Theorem 3.2. Let $A$ be a finite group, and let $G$ be a connected Lie group with an $A$-module structure. Then we have

(i) $Z^{1}(A, G) \subset \operatorname{Map}(A, G)$ has finitely many connected components, each of which is a closed submanifold of $\operatorname{Map}(A, G)$;

(ii) Each cohomology class in $Z^{1}(A, G)$ is a connected component of $Z^{1}(A, G)$, that is, $H^{1}(A, G)$ coincides with the set of all connected components of $Z^{1}(A, G)$.

The following fact is needed in the proof of Theorem 3.2. For a proof of it, see [14], Part II, Chapter III, Section 11.

Fact 3.2 (Weil's Lemma). Let $M, N$ be smooth manifolds, and let $\varphi: M \rightarrow N$ be a smooth map. Let $x \in N$. Suppose that for every $y \in \varphi^{-1}(x)$, there exist a smooth manifold $L$, a smooth map $\psi: L \rightarrow M$, and a point $z \in L$ such that

(i) $\psi(z)=y$;

(ii) $\varphi(\psi(w))=x$ for all $w \in L$;

(iii) The sequence $T_{z} L \stackrel{(d \psi)_{z}}{\longrightarrow} T_{y} M \stackrel{(d \varphi)_{y}}{\longrightarrow} T_{x} N$ is exact.

Then every connected component of $\varphi^{-1}(x)$ is a closed submanifold of $M$. Moreover, there exist a neighborhood $U$ of $y$ in $M$ and a neighborhood $V$ of $z$ in $L$ such that $\psi(V)=U \cap \varphi^{-1}(x)$.

Proof of Theorem 3.2. Let $\mathfrak{g}$ be the Lie algebra of $G$. Then for $\alpha \in \operatorname{Map}(A, G)$, the tangent space $T_{\alpha} \operatorname{Map}(A, G)$ of $\operatorname{Map}(A, G)$ at $\alpha$ can be naturally identified with $\operatorname{Map}(A, \mathfrak{g})$, by the correspondence that $\lambda \in \operatorname{Map}(A, \mathfrak{g})$ is mapped to $\left.\frac{d}{d t}\right|_{t=0} e^{t \lambda} \alpha \in$ $T_{\alpha} \operatorname{Map}(A, G)$. Equivalently, for a smooth curve $\gamma: \mathbb{R} \rightarrow \operatorname{Map}(A, G)$ with $\gamma(0)=$ $\alpha$, the tangent vector $\left.\frac{d}{d t}\right|_{t=0} \gamma(t)$ corresponds to the element of $\operatorname{Map}(A, \mathfrak{g})$ defined by $\left.a \mapsto \frac{d}{d t}\right|_{t=0} \gamma(t)(a) \alpha(a)^{-1}$. Similarly, tangent spaces of $\operatorname{Map}\left(A^{2}, G\right)$ may be identified with $\operatorname{Map}\left(A^{2}, \mathfrak{g}\right)$.

We first prove that every connected component of $Z^{1}(A, G) \subset \operatorname{Map}(A, G)$ is a closed submanifold. Define a map $\varphi: \operatorname{Map}(A, G) \rightarrow \operatorname{Map}\left(A^{2}, G\right)$ by $\varphi(\alpha)(a, b)=$ $\alpha(a) a(\alpha(b)) \alpha(a b)^{-1}$. Denote the constant map $(a, b) \mapsto e$ in $\operatorname{Map}\left(A^{2}, G\right)$ also by $e$. Then $\varphi^{-1}(e)=Z^{1}(A, G)$. Now for $\alpha\left(a \mapsto g_{a}\right) \in Z^{1}(A, G)$, we compute $\operatorname{ker}\left((d \varphi)_{\alpha}\right)$. For $\lambda\left(a \mapsto X_{a}\right) \in \operatorname{Map}(A, \mathfrak{g}) \cong T_{\alpha} \operatorname{Map}(A, G)$ and $a, b \in A$, we have

$$
\begin{aligned}
& (d \varphi)_{\alpha}(\lambda)(a, b) \\
= & (d \varphi)_{\alpha}\left(\left.\frac{d}{d t}\right|_{t=0} e^{t \lambda} \alpha\right)(a, b) \\
= & \left.\frac{d}{d t}\right|_{t=0} \varphi\left(e^{t \lambda} \alpha\right)(a, b) \\
= & \left.\frac{d}{d t}\right|_{t=0}\left(e^{t X_{a}} g_{a}\right) a\left(e^{t X_{b}} g_{b}\right)\left(e^{t X_{a b}} g_{a b}\right)^{-1} \\
= & \left.\frac{d}{d t}\right|_{t=0} e^{t X_{a}}\left(g_{a} e^{t d a\left(X_{b}\right)} a\left(g_{b}\right) g_{a b}^{-1}\right) e^{-t X_{a b}} \\
= & \left.\frac{d}{d t}\right|_{t=0} e^{t X_{a}} e^{t \operatorname{Ad}\left(g_{a}\right) d a\left(X_{b}\right)} e^{-t X_{a b}} \\
= & X_{a}+\operatorname{Ad}\left(g_{a}\right) d a\left(X_{b}\right)-X_{a b} .
\end{aligned}
$$

So $\lambda\left(a \mapsto X_{a}\right) \in \operatorname{ker}\left((d \varphi)_{\alpha}\right)$ if and only if $X_{a b}=X_{a}+\operatorname{Ad}\left(g_{a}\right) d a\left(X_{b}\right)$ for all $a, b \in A$. Since $\operatorname{Ad}\left(g_{a}\right) d a$ is the differential of the action of $a$ on $G_{\alpha}$, the $A$-module 
obtained by twisting $G$ using $\alpha, a \mapsto \operatorname{Ad}\left(g_{a}\right) d a$ is a linear action of $A$ on $\mathfrak{g}$. Hence $\operatorname{ker}\left((d \varphi)_{\alpha}\right)=Z^{1}(A, \mathfrak{g})$.

For $\alpha \in Z^{1}(A, G)$ in the previous paragraph, we define $\psi: G \rightarrow \operatorname{Map}(A, G)$ by $\psi(g)(a)=g^{-1} g_{a} a(g)$. Then $\psi(e)=\alpha$ and $\psi(g) \in Z^{1}(A, G)$ for all $g \in G$. For $X \in \mathfrak{g}$, we have, under the identification $T_{\alpha} \operatorname{Map}(A, G) \cong \operatorname{Map}(A, \mathfrak{g})$,

$$
\begin{aligned}
& (d \psi)_{e}(X)(a) \\
= & \left.\frac{d}{d t}\right|_{t=0} e^{-t X} g_{a} a\left(e^{t X}\right) g_{a}^{-1} \\
= & \left.\frac{d}{d t}\right|_{t=0} e^{-t X} e^{t \operatorname{Ad}\left(g_{a}\right) d a(X)} \\
= & -X+\operatorname{Ad}\left(g_{a}\right) d a(X) .
\end{aligned}
$$

Hence $\operatorname{Im}\left((d \psi)_{e}\right)=B^{1}(A, \mathfrak{g})$, the set of 1-coboundaries with respect to the linear action of $A$ on $\mathfrak{g}$ mentioned above.

Note that $H^{1}(A, \mathfrak{g})=Z^{1}(A, \mathfrak{g}) / B^{1}(A, \mathfrak{g})$ is a linear space over $\mathbb{R}$. But by [12], Chapter VIII, Section 2, Corollary 1, all elements of $H^{1}(A, \mathfrak{g})$ are annihilated by $|A|$, the order of $A$. So $H^{1}(A, \mathfrak{g})=0$, that is, $Z^{1}(A, \mathfrak{g})=B^{1}(A, \mathfrak{g})$, and then $\operatorname{ker}\left((d \varphi)_{\alpha}\right)=\operatorname{Im}\left((d \psi)_{e}\right)$. By Weil's Lemma, every connected component of $Z^{1}(A, G)=\varphi^{-1}(e)$ is a closed submanifold of $\operatorname{Map}(A, G)$.

Now we prove that every cohomology class in $Z^{1}(A, G)$ is a connected component of $Z^{1}(A, G)$. In fact, for $\alpha \in Z^{1}(A, G)$, the cohomology class of $\alpha$ is the image of the map $\psi$ defined above. By Weil's Lemma, there exist a neighborhood $U$ of $\alpha$ in $\operatorname{Map}(A, G)$ and a neighborhood $V$ of $e$ in $G$ such that $\psi(V)=U \cap Z^{1}(A, G)$. So the cohomology class of $\alpha$ is open in $Z^{1}(A, G)$. Since the cohomology class of $\alpha$ is the complement of the union of other cohomology classes, which are all open in $Z^{1}(A, G)$, it is also closed in $Z^{1}(A, G)$. But as the image of $\psi$, it is connected. Hence it is a connected component of $Z^{1}(A, G)$.

Finally we prove that $Z^{1}(A, G)$ has only finitely many connected components. Since we have proved that the cardinality of the set of connected components of $Z^{1}(A, G)$ coincides with that of $H^{1}(A, G)$, by Theorem 3.1 , it is sufficient to prove the case for which $G$ is compact. But if $G$ is compact, so is $\operatorname{Map}(A, G)$, and as we have showed above, every connected component of the closed subset $Z^{1}(A, G)$ of $\operatorname{Map}(A, G)$ is open in $Z^{1}(A, G)$. This forces that $Z^{1}(A, G)$ has only finitely many connected components. The proof of the theorem is finished.

Corollary 3.1. Let $A$ be a finite group, and let $G$ be a connected Lie group with an $A$-module structure. Then $H^{1}(A, G)$ is finite.

Remark 3.1. Professor Jiu-Kang Yu had a simple algebro-geometric proof of Theorem 1.2 when $G$ is compact.

\section{Nonabelian COHOMOlogy of $\mathbb{Z}$ With COEFFicients in Lie Groups}

Let $V$ be a finite-dimensional real vector space, and let $L$ be a linear endomorphism of $V$. For a complex number $\lambda, L$ is $\lambda$-semisimple if $\operatorname{ker}(\lambda I-L)=$ $\operatorname{ker}\left((\lambda I-L)^{2}\right)$ in the complexification $V_{\mathbb{C}}$ of $V$. Note that $L$ is semisimple if and only if it is $\lambda$-semisimple for every $\lambda \in \mathbb{C}$. For a connected Lie group $G$ and a complex number $\lambda$, an automorphism $\sigma$ of $G$ is $\lambda$-semisimple (semisimple, resp.) if the linear endomorphism $d \sigma$ on the Lie algebra $\mathfrak{g}$ of $G$ is $\lambda$-semisimple (semisimple, resp.). Suppose $A$ is a cyclic group and $G$ is a connected Lie group with an 
$A$-module structure. The action of $A$ on $G$ is 1-semisimple (semisimple, resp.) if the action of a generator of $A$ on $G$ is 1-semisimple (semisimple, resp.). Note that this definition is independent of the choice of the generator of $A$.

All automorphisms of a connected compact semisimple Lie group are 1-semisimple, due to the fact that the automorphism group of a compact semisimple Lie group is compact. On the other hand, not all automorphisms of a compact torus of dimension $\geq 2$ are 1-semisimple. For example, the automorphism of $\mathbb{R}^{2} / \mathbb{Z}^{2}$ induced by $\left(\begin{array}{ll}1 & 1 \\ 0 & 1\end{array}\right)$ is not 1-semisimple. In fact, for a connected Lie group $G$ with an automorphism $\sigma, \sigma$ is 1-semisimple if and only if the restriction of $\sigma$ to the connected component of the center of $G$ is 1-semisimple.

The main content of this section is to prove the following theorem.

Theorem 4.1. Let $G$ be a connected compact Lie group with a $\mathbb{Z}$-module structure, and let $T$ be a maximal torus of $G_{0}^{\mathbb{Z}}$. Let $i_{1}: H^{1}(\mathbb{Z}, T) \rightarrow H^{1}(\mathbb{Z}, G)$ be the canonical map. Then the following statements are equivalent:

(i) The $\mathbb{Z}$-action on $G$ is 1-semisimple;

(ii) The map $i_{1}$ is surjective;

(iii) The kernel of $i_{1}$ is finite;

(iv) All fibers of $i_{1}$ are finite.

To prove Theorem 4.1, we need several lemmas. The first lemma is a key step, whose proof needs the following facts.

Fact 4.1 ([7], Chapter II, Theorem 5.1). Let $M$ be a Riemannian manifold with an isometry $f$. Then every connected component of $\operatorname{Fix}(f)$ is a closed totally geodesic submanifold of $M$, where $\operatorname{Fix}(f)$ is the set of fixed points of $f$.

Fact 4.2 ([7], Chapter II, Section 5, page 63). Let $M$ be a compact Riemannian manifold with an isometry $f$. Then $\operatorname{Lef}(f)=\chi(\operatorname{Fix}(f))$, where $\operatorname{Lef}(f)$ is the Lefschetz number of $f$ and $\chi(\operatorname{Fix}(f))$ is the Euler number of $\operatorname{Fix}(f)$.

Fact 4.3 ([8], Chapter VII, Corollary 8.10). Let $M$ be a compact homogeneous Riemannian manifold. Then every closed totally geodesic submanifold of $M$ is a compact homogeneous Riemannian manifold.

Fact 4.4 ([11], Theorem II). Let $N$ be a connected compact homogeneous Riemannian manifold. Then $\chi(N) \geq 0$. Write $N$ as the form $N=K / H$, where $K$ and $H$ are compact Lie groups and $K$ is connected. Then $\chi(N)>0$ if and only if $\operatorname{rank}(K)=\operatorname{rank}\left(H_{0}\right)$.

Lemma 4.1. Let $G$ be a connected compact semisimple Lie group with a $\mathbb{Z}$-module structure and $T$ a maximal torus of $G_{0}^{\mathbb{Z}}$. Then the canonical map $H^{1}(\mathbb{Z}, T) \rightarrow$ $H^{1}(\mathbb{Z}, G)$ is surjective.

Sublemma 4.1.1. Suppose $G$ is a connected compact semisimple Lie group with a closed subgroup $H$. Denote $\operatorname{Aut}(G, H)=\{\theta \in \operatorname{Aut}(G) \mid \theta(H)=H\}$, which acts on $G / H$ by $\rho_{\theta}(g H)=\theta(g) H$. Then there is an $\operatorname{Ad}(G)$-invariant inner product on $\mathfrak{g}$ such that the induced $G$-invariant Riemannian structure on $G / H$ is also $\operatorname{Aut}(G, H)$ invariant.

Proof. We construct the semidirect product $G \rtimes \operatorname{Aut}(G, H)$, and define the map $\varphi:(G \rtimes \operatorname{Aut}(G, H)) \times G / H \rightarrow G / H$ by $\varphi\left((g, \theta), g^{\prime} H\right)=g \theta\left(g^{\prime}\right) H$. We claim that 
$\varphi$ is an action of $G \rtimes \operatorname{Aut}(G, H)$ on $G / H$. In fact, if we denote $\varphi_{(g, \theta)}=\varphi((g, \theta), \cdot)$, then

$$
\begin{aligned}
& \varphi_{\left(g_{1}, \theta_{1}\right)} \circ \varphi_{\left(g_{2}, \theta_{2}\right)}\left(g^{\prime} H\right) \\
= & \varphi_{\left(g_{1}, \theta_{1}\right)}\left(g_{2} \theta_{2}\left(g^{\prime}\right) H\right) \\
= & g_{1} \theta_{1}\left(g_{2}\right) \theta_{1}\left(\theta_{2}\left(g^{\prime}\right)\right) H \\
= & \varphi_{\left(g_{1} \theta_{1}\left(g_{2}\right), \theta_{1} \circ \theta_{2}\right)}\left(g^{\prime} H\right) \\
= & \varphi_{\left(g_{1}, \theta_{1}\right)\left(g_{2}, \theta_{2}\right)}\left(g^{\prime} H\right) .
\end{aligned}
$$

This verifies the claim. Note that the action of $G \rtimes \operatorname{Aut}(G, H)$ on $G / H$ is transitive, and the isotropic subgroup associated with the point $H \in G / H$ is $H \rtimes$ $\operatorname{Aut}(G, H)$. So there is a natural isomorphism $G / H \cong G \rtimes \operatorname{Aut}(G, H) / H \rtimes$ $\operatorname{Aut}(G, H)$. Since $G$ is compact semisimple, $\operatorname{Aut}(G)$ is compact. As a closed subgroup of $\operatorname{Aut}(G), \operatorname{Aut}(G, H)$ is also compact. So $G \rtimes \operatorname{Aut}(G, H)$ is compact. Choose an $\operatorname{Ad}(G \rtimes \operatorname{Aut}(G, H))$-invariant inner product $B(\cdot, \cdot)$ on the Lie algebra

$$
\mathrm{L}(G \rtimes \operatorname{Aut}(G, H)) \text { of } G \rtimes \operatorname{Aut}(G, H) .
$$

Then the restriction of $B$ on $\mathrm{L}(H \rtimes \operatorname{Aut}(G, H))^{\perp}$ induces a $G \rtimes \operatorname{Aut}(G, H)$-invariant Riemannian structure on $G / H$. Note that if we identify $G \times\{i d\}$ with $G$ and $\{e\} \times \operatorname{Aut}(G, H)$ with $\operatorname{Aut}(G, H)$, the restrictions of the $G \rtimes \operatorname{Aut}(G, H)$-action to $G$ and $\operatorname{Aut}(G, H)$ coincide with the natural actions of $G$ and $\operatorname{Aut}(G, H)$ on $G / H$. So the $G \rtimes \operatorname{Aut}(G, H)$-invariant Riemannian structure on $G / H$ constructed above is $G$-invariant and $\operatorname{Aut}(G, H)$-invariant.

Note that the restriction of the inner product $B$ on the Lie algebra $\mathfrak{g}$ of $G$ is $\operatorname{Ad}(G)$-invariant, and the restriction of $B$ on $\mathfrak{h}^{\perp}$ induces a $G$-invariant Riemannian structure on $G / H$ which is the same as the one constructed above. This proves the sublemma.

Sublemma 4.1.2. Under the same conditions as in Sublemma 4.1.1, if $\theta \in$ $\operatorname{Aut}(G, H)$ satisfies $H \subset G^{\theta}$, then the connected component of $\operatorname{Fix}\left(\rho_{\theta}\right) \subset G / H$ containing the point $H$ is $G_{0}^{\theta} / H$.

Proof. Endow $G / H$ with a $G$-invariant and an $\operatorname{Aut}(G, H)$-invariant Riemannian structure which is induced by an $\operatorname{Ad}(G)$-invariant inner product on $\mathfrak{g}$. By Corollary 3.6, Theorem 2.10, and Corollary 2.5 in Chapter X of [8], every geodesic of $G / H$ starting from $H$ is of the form $e^{t X} H$ for some $X \in \mathfrak{g}$.

Denote the connected component of $\operatorname{Fix}\left(\rho_{\theta}\right)$ containing $H$ by $F_{0}$. By Fact 4.1, $F_{0}$ is a closed totally geodesic submanifold of $G / H$. Suppose $g H \in F_{0}$. Let $e^{t X} H$ be a geodesic through $H$ and $g H$ such that $e^{t X} H \in F_{0}, \forall t \in \mathbb{R}$. Then $e^{t X} H=$ $\rho_{\theta}\left(e^{t X} H\right)=\theta\left(e^{t X}\right) H=e^{t d \theta(X)} H$. This implies $e^{-t X} e^{t d \theta(X)} \in H$. So $(d \theta-1)(X) \in$ $\mathfrak{h} \subset \mathrm{L}\left(G^{\theta}\right)=\operatorname{ker}(d \theta-1)$. Since $G$ is compact semisimple, $d \theta$ is semisimple. This implies $X \in \mathrm{L}\left(G^{\theta}\right)$, and then $g H \in G_{0}^{\theta} / H$. So $F_{0} \subset G_{0}^{\theta} / H$. The inverse direction is obvious.

Proof of Lemma 4.1. Let $\sigma$ be a generator of $\mathbb{Z}$. Then under the identification $Z^{1}(\mathbb{Z}, G) \cong G$ (see Section 2 ), two cocycles $z, z^{\prime} \in G$ are cohomologous if there exists $g \in G$ such that $z^{\prime}=g^{-1} z \sigma(g)$. So to prove the lemma, it is sufficient to show that for each $z \in G$, there exists $g \in G$ such that $g^{-1} z \sigma(g) \in T$. 
Let $f$ be the diffeomorphism of $G / T$ defined by $f(g T)=\sigma(g) T$. Since $\sigma \in$ $\operatorname{Aut}(G, T)$ and $T \subset G^{\mathbb{Z}}$, by Sublemma 4.1.1 and Sublemma 4.1.2, there is a $G$ invariant Riemannian structure on $G / T$ which is also $f$-invariant, and the connected component of Fix $(f)$ containing $T$ is $G_{0}^{\mathbb{Z}} / T$. Let $\left\{F_{0}, F_{1}, \cdots\right\}$ be the set of all the connected components of $\operatorname{Fix}(f)$ with $F_{0}=G_{0}^{\mathbb{Z}} / T$. Since $\operatorname{rank}\left(G_{0}^{\mathbb{Z}}\right)=\operatorname{rank}(T)$, by Fact 4.4 , we have $\chi\left(G_{0}^{\mathbb{Z}} / T\right)>0$. Since $f$ is an isometry with respect to the abovementioned Riemannian structure on $G / T$, by Fact 4.1 , each $F_{i}$ is a closed totally geodesic submanifold of $G / T$. Then by Fact 4.3 , each $F_{i}$ is a compact homogeneous Riemannian manifold. So $\chi\left(F_{i}\right) \geq 0$ for each $i$, by Fact 4.4. Applying the formula in Fact 4.2, we have

$$
\operatorname{Lef}(f)=\chi(\operatorname{Fix}(f))=\sum_{i \geq 0} \chi\left(F_{i}\right)=\chi\left(G_{0}^{\mathbb{Z}} / T\right)+\sum_{i>0} \chi\left(F_{i}\right) \geq \chi\left(G_{0}^{\mathbb{Z}} / T\right)>0 .
$$

For each $z \in G$, define the diffeomorphism $f_{z}$ of $G / T$ by $f_{z}(g T)=z \sigma(g) T$. Since $G$ is connected, $f_{z}$ is homotopic to $f=f_{e}$, and then $\operatorname{Lef}\left(f_{z}\right)=\operatorname{Lef}(f)>0$. By the Lefschetz Fixed Point Theorem, $f_{z}$ has a fixed point $g T$, that is, $z \sigma(g) T=g T$. This means that $g^{-1} z \sigma(g) \in T$. The lemma is proved.

Lemma 4.2. Let $G$ be a compact torus with a $\mathbb{Z}$-module structure, $T=G_{0}^{\mathbb{Z}}$.

(i) If the $\mathbb{Z}$-action on $G$ is 1 -semisimple, then $H^{1}(\mathbb{Z}, T) \rightarrow H^{1}(\mathbb{Z}, G)$ is surjective.

(ii) If there exists a discrete $\mathbb{Z}$-submodule $\Gamma$ of $G$ such that $H^{1}(\mathbb{Z}, T \Gamma) \rightarrow H^{1}(\mathbb{Z}, G)$ is surjective, then the $\mathbb{Z}$-action on $G$ is 1 -semisimple.

Proof. (i) We also choose a generator $\sigma$ of $\mathbb{Z}$ and identify $Z^{1}(\mathbb{Z}, G)$ with $G$. Let $z \in G$ be a cocycle, and choose an $X \in \mathfrak{g}$ such that $e^{X}=z$. Since $\sigma$ is 1 -semisimple, $\operatorname{ker}(1-d \sigma) \oplus \operatorname{Im}(1-d \sigma)=\mathfrak{g}$. So we can write $X$ as $X=Y+(1-d \sigma)(Z)$, that is, $-Z+X+d \sigma(Z)=Y$, where $Y \in \operatorname{ker}(1-d \sigma), Z \in \mathfrak{g}$. Since $G$ is abelian, this implies that $e^{-Z} z \sigma\left(e^{Z}\right)=e^{Y} \in T$. This proves (i).

(ii) We prove it by contradiction. Suppose that the $\mathbb{Z}$-action on $G$ is not 1 semisimple. So $\operatorname{ker}(1-d \sigma)+\operatorname{Im}(1-d \sigma) \varsubsetneqq \mathfrak{g}$. Then for each discrete $\mathbb{Z}$-submodule $\Gamma$ of $G, \operatorname{ker}(1-d \sigma)+\operatorname{Im}(1-d \sigma)+\exp ^{-1}(\Gamma) \varsubsetneqq \mathfrak{g}$. Choose an $X \in \mathfrak{g}$ which does not belong to the left hand side of the above equation. Then it is easy to check that for every $g \in G, g^{-1} e^{X} \sigma(g) \notin T \Gamma$, that is, $H^{1}(\mathbb{Z}, T \Gamma) \rightarrow H^{1}(\mathbb{Z}, G)$ is not surjective. This conflicts with the conditions, and then (ii) is proved.

Lemma 4.3. Let $G, G^{\prime}$ be connected Lie groups with $\mathbb{Z}$-module structures, and let $\pi: G^{\prime} \rightarrow G$ be a $\mathbb{Z}$-epimorphism.

(i) If $H^{\prime}$ is a submodule of $G^{\prime}$ such that $H^{1}\left(\mathbb{Z}, H^{\prime}\right) \rightarrow H^{1}\left(\mathbb{Z}, G^{\prime}\right)$ is surjective, then $H^{1}\left(\mathbb{Z}, \pi\left(H^{\prime}\right)\right) \rightarrow H^{1}(\mathbb{Z}, G)$ is surjective.

(ii) If $H$ is a submodule of $G$ such that $H^{1}(\mathbb{Z}, H) \rightarrow H^{1}(\mathbb{Z}, G)$ is surjective, then $H^{1}\left(\mathbb{Z}, \pi^{-1}(H)\right) \rightarrow H^{1}\left(\mathbb{Z}, G^{\prime}\right)$ is surjective.

Proof. The first assertion follows from the following commutative diagram, in which the two columns are surjective:

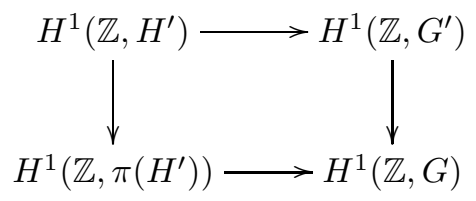


To prove the second assertion, let $z^{\prime} \in G^{\prime}$ be a cocycle. If $H^{1}(\mathbb{Z}, H) \rightarrow H^{1}(\mathbb{Z}, G)$ is surjective, then there exists $g \in G$ such that $g^{-1} \pi\left(z^{\prime}\right) \sigma(g) \in H$. Choose a $g^{\prime} \in G^{\prime}$ with $\pi\left(g^{\prime}\right)=g$; then $\pi\left(g^{\prime-1} z^{\prime} \sigma\left(g^{\prime}\right)\right)=g^{-1} \pi\left(z^{\prime}\right) \sigma(g) \in H$, that is, $g^{\prime-1} z^{\prime} \sigma\left(g^{\prime}\right) \in$ $\pi^{-1}(H)$. Hence $H^{1}\left(\mathbb{Z}, \pi^{-1}(H)\right) \rightarrow H^{1}\left(\mathbb{Z}, G^{\prime}\right)$ is surjective.

Lemma 4.4. Let $G$ be a connected compact semisimple Lie group with a $\mathbb{Z}$-module structure, and let $T$ be a maximal torus of $G_{0}^{\mathbb{Z}}$. Then all fibers of $H^{1}(\mathbb{Z}, T) \rightarrow$ $H^{1}(\mathbb{Z}, G)$ are finite.

Sublemma 4.4.1. Let $M$ be a compact smooth manifold with two closed smooth submanifolds $N_{1}, N_{2}$. If for each $p \in N_{1} \cap N_{2}, T_{p} N_{1} \cap T_{p} N_{2}=0$, then $N_{1} \cap N_{2}$ is a finite set.

Proof. Let $p \in N_{1} \cap N_{2}$. Choose a coordinate chart $\left(U, x_{1}, \cdots, x_{n}\right)$ of $M$ containing $p$ such that $x_{i}(p)=0,1 \leq i \leq n$, and such that $U \cap N_{1}=\left\{q \in U \mid x_{n_{1}+1}(q)=\right.$ $\left.\cdots=x_{n}(q)=0\right\}$, where $n=\operatorname{dim} M, n_{1}=\operatorname{dim} N_{1}$. Define $f: U \rightarrow \mathbb{R}^{n-n_{1}}$ by $f(q)=\left(x_{n_{1}+1}(q), \cdots, x_{n}(q)\right)$. Then $T_{p} N_{1} \cap T_{p} N_{2}=0$ implies that $\left.f\right|_{U \cap N_{2}}$ : $U \cap N_{2} \rightarrow \mathbb{R}^{n-n_{1}}$ is an immersion at $p$. Hence there is an open neighborhood $V \subset U \cap N_{2}$ of $p$ in $N_{2}$ such that $\left.f\right|_{V}$ is injective. In particular, $V \cap N_{1}=\{p\}$. This proves that $N_{1} \cap N_{2}$ is discrete in $N_{2}$. Since $N_{2}$ is compact, $N_{1} \cap N_{2}$ is finite.

Proof of Lemma 4.4. A fiber of $H^{1}(\mathbb{Z}, T) \rightarrow H^{1}(\mathbb{Z}, G)$ is the intersection of $T$ with a cohomology class in $Z^{1}(\mathbb{Z}, G)$. But a cohomology class in $Z^{1}(\mathbb{Z}, G)$ is an orbit of the twisted conjugate action $\tau$ associated with $\sigma$ (see Section 2), which is a closed submanifold. So by Sublemma 4.4.1, it is sufficient to prove that for each $t \in T$, $T_{t} O_{t} \cap T_{t} T=0$, where $O_{t}$ is the orbit of the twisted conjugate action containing $t$.

Let $l_{t}$ be the left translation on $G$ induced by $t$. Since

$$
\begin{aligned}
& \left.\frac{d}{d s}\right|_{s=0} \tau_{e^{s X}}(t) \\
= & \left(d l_{t}\right)_{e}\left(\left.\frac{d}{d s}\right|_{s=0} t^{-1} e^{s X} t \sigma\left(e^{s X}\right)^{-1}\right) \\
= & \left(d l_{t}\right)_{e}\left(\left.\frac{d}{d s}\right|_{s=0} e^{s \operatorname{Ad}\left(t^{-1}\right) X} e^{-s d \sigma(X)}\right) \\
= & \left(d l_{t}\right)_{e}\left(\operatorname{Ad}\left(t^{-1}\right)-d \sigma\right)(X),
\end{aligned}
$$

we have $T_{t} O_{t} \cap T_{t} T=\left(d l_{t}\right)_{e}\left(\operatorname{Im}\left(\operatorname{Ad}\left(t^{-1}\right)-d \sigma\right) \cap \mathfrak{t}\right)$, and it is sufficient to show that $\operatorname{Im}\left(\operatorname{Ad}\left(t^{-1}\right)-d \sigma\right) \cap \mathfrak{t}=0$. Since $G$ is compact semisimple, $\operatorname{Ad}\left(t^{-1}\right)$ and $d \sigma$ are semisimple. The fact that $\sigma(t)=t$ implies that $\operatorname{Ad}\left(t^{-1}\right)$ and $d \sigma$ commute, so $\operatorname{Ad}\left(t^{-1}\right)-d \sigma$ is also semisimple. But $\mathfrak{t} \subset \operatorname{ker}\left(\operatorname{Ad}\left(t^{-1}\right)-d \sigma\right)$, so $\operatorname{Im}\left(\operatorname{Ad}\left(t^{-1}\right)-d \sigma\right) \cap$ $\mathfrak{t}=0$. This proves the lemma.

Lemma 4.5. Let $G$ be a compact torus with a $\mathbb{Z}$-module structure, $T=G_{0}^{\mathbb{Z}}$. Then the kernel of $i_{1}: H^{1}(\mathbb{Z}, T) \rightarrow H^{1}(\mathbb{Z}, G)$ is finite if and only if the $\mathbb{Z}$-action on $G$ is 1-semisimple. 
Proof. Since $G$ is abelian, we have the following exact sequence of cohomology groups:

$$
0 \rightarrow H^{0}(\mathbb{Z}, T) \stackrel{i_{0}}{\rightarrow} H^{0}(\mathbb{Z}, G) \rightarrow H^{0}(\mathbb{Z}, G / T) \stackrel{\delta}{\rightarrow} H^{1}(\mathbb{Z}, T) \stackrel{i_{1}}{\rightarrow} H^{1}(\mathbb{Z}, G) .
$$

Since $\operatorname{ker}(\delta) \cong \operatorname{coker}\left(i_{0}\right)=G^{\mathbb{Z}} / T$ is finite,

$$
\begin{aligned}
& \operatorname{ker}\left(i_{1}\right)=\operatorname{Im}(\delta) \text { is finite } \\
\Leftrightarrow & H^{0}(\mathbb{Z}, G / T) \text { is finite } \\
\Leftrightarrow & \text { the induced linear automorphism of } d \sigma \text { on } \mathfrak{g} / \mathfrak{t} \text { has no eigenvalue } 1 \\
\Leftrightarrow & d \sigma \text { is } 1 \text {-semisimple } \\
\Leftrightarrow & \text { the } \mathbb{Z} \text {-action on } G \text { is } 1 \text {-semisimple. }
\end{aligned}
$$

Proof of Theorem 4.1. We follow the line "(i) $\Rightarrow($ ii $) \Rightarrow($ i $) \Rightarrow($ iv $) \Rightarrow($ iii $) \Rightarrow$ (i)".

Let $\mathfrak{g}_{s}=[\mathfrak{g}, \mathfrak{g}], \mathfrak{g}_{t}$ be the center of $\mathfrak{g}$. Then $\mathfrak{g}=\mathfrak{g}_{s} \oplus \mathfrak{g}_{t}$. Let $G_{s}, G_{t}$ be the connected subgroups of $G$ with Lie algebras $\mathfrak{g}_{s}$ and $\mathfrak{g}_{t}$, respectively. Then $G_{s}$ is semisimple, $G_{t}$ is a compact torus, and it is obvious that $G_{s}$ and $G_{t}$ are $\mathbb{Z}$ submodules of $G$. Let $G^{\prime}=G_{s} \times G_{t}$ be the direct product of $\mathbb{Z}$-modules, and define $\pi: G^{\prime} \rightarrow G$ by $\pi\left(g_{s}, g_{t}\right)=g_{s} g_{t}$. Then $\pi$ is a $\mathbb{Z}$-epimorphism which is also a finite covering homomorphism of Lie groups. Let $T_{s}=\left(T \cap G_{s}\right)_{0}, T_{t}=\left(T \cap G_{t}\right)_{0}, T^{\prime}=$ $T_{s} \times T_{t}$. Then $T_{s}, T_{t}$, and $T^{\prime}$ are maximal tori of $\left(G_{s}^{\mathbb{Z}}\right)_{0},\left(G_{t}^{\mathbb{Z}}\right)_{0}$, and $G_{0}^{\prime \mathbb{Z}}$, respectively, and we have $\pi\left(T^{\prime}\right)=T$. Note that the $\mathbb{Z}$-action on $G$ is 1 -semisimple if and only if the $\mathbb{Z}$-action on $G_{t}$ is 1 -semisimple.

"(i) $\Rightarrow($ ii)". Suppose that the $\mathbb{Z}$-action on $G$ is 1 -semisimple. By Lemma 4.1 and part (i) of Lemma $4.2, H^{1}\left(\mathbb{Z}, T_{s}\right) \rightarrow H^{1}\left(\mathbb{Z}, G_{s}\right)$ and $H^{1}\left(\mathbb{Z}, T_{t}\right) \rightarrow H^{1}\left(\mathbb{Z}, G_{t}\right)$ are surjective. From the commutative diagram

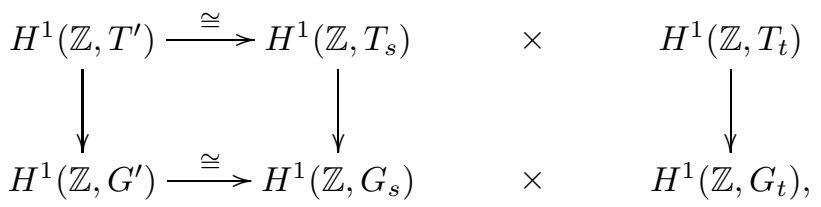

we know that $H^{1}\left(\mathbb{Z}, T^{\prime}\right) \rightarrow H^{1}\left(\mathbb{Z}, G^{\prime}\right)$ is surjective. By part (i) of Lemma 4.3, $H^{1}(\mathbb{Z}, T) \rightarrow H^{1}(\mathbb{Z}, G)$ is surjective.

"(ii) $\Rightarrow\left(\right.$ i)". Suppose that $H^{1}(\mathbb{Z}, T) \rightarrow H^{1}(\mathbb{Z}, G)$ is surjective. By part (ii) of Lemma $4.3, H^{1}\left(\mathbb{Z}, T^{\prime} \cdot \operatorname{ker}(\pi)\right) \rightarrow H^{1}\left(\mathbb{Z}, G^{\prime}\right)$ is surjective. Applying part (i) of Lemma 4.3 to the projection $p_{2}: G^{\prime}=G_{s} \times G_{t} \rightarrow G_{t}$, we know that

$$
H^{1}\left(\mathbb{Z}, T_{t} \cdot p_{2}(\operatorname{ker}(\pi))\right) \rightarrow H^{1}\left(\mathbb{Z}, G_{t}\right)
$$

is surjective. But $p_{2}(\operatorname{ker}(\pi))$ is a discrete $\mathbb{Z}$-submodule of $G_{t}$; by part (ii) of Lemma 4.2 , the $\mathbb{Z}$-action on $G_{t}$ is 1 -semisimple. Hence the $\mathbb{Z}$-action on $G$ is 1 -semisimple.

"(i) $\Rightarrow$ (iv)". Suppose that the $\mathbb{Z}$-action on $G$ is 1 -semisimple. We show that all fibers of $H^{1}(\mathbb{Z}, T) \rightarrow H^{1}(\mathbb{Z}, G)$ are finite. First we prove the kernel of this map is finite. By Lemma 4.4, Lemma 4.5, and the commutative diagram (4.1), we know that the kernel of $H^{1}\left(\mathbb{Z}, T^{\prime}\right) \rightarrow H^{1}\left(\mathbb{Z}, G^{\prime}\right)$ is finite. Since $\pi: G^{\prime} \rightarrow G$ is a finite cover, $\operatorname{ker}(\pi)$ and $\operatorname{ker}\left(\left.\pi\right|_{T^{\prime}}\right)$ are finite. Hence $H^{1}(\mathbb{Z}, \operatorname{ker}(\pi))$ and $H^{1}\left(\mathbb{Z}, \operatorname{ker}\left(\left.\pi\right|_{T^{\prime}}\right)\right)$ 
are finite. By the commutative diagram

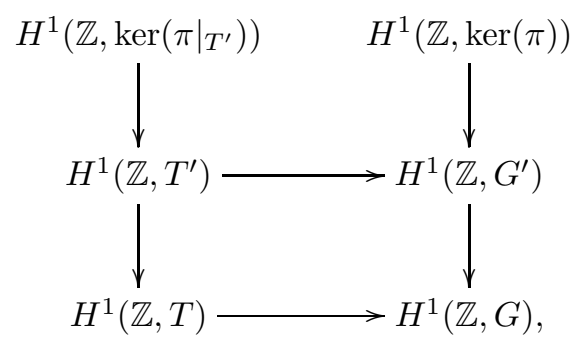

in which the two columns are exact, we know that the kernel of $H^{1}(\mathbb{Z}, T) \rightarrow$ $H^{1}(\mathbb{Z}, G)$ is finite.

Now let $[\alpha] \in H^{1}(\mathbb{Z}, G)$. By the step "(i) $\Rightarrow($ ii $)$ " above, we know the fiber $i_{1}^{-1}([\alpha])$ is not empty. Choose a $[\beta] \in i_{1}^{-1}([\alpha])$, where $\beta \in[\beta]$. Then the twisting using $\beta$ transforms the fiber $i_{1}^{-1}([\alpha])$ to the kernel of $H^{1}\left(\mathbb{Z}, T_{\beta}\right) \rightarrow H^{1}\left(\mathbb{Z}, G_{i \circ \beta}\right)$ (see Section $2)$. Since $T_{\beta} \subset\left(G_{i \circ \beta}^{\mathbb{Z}}\right)_{0}$, we can choose a maximal torus $T_{\beta}^{\prime}$ of $\left(G_{i \circ \beta}^{\mathbb{Z}}\right)_{0}$ with $T_{\beta} \subset T_{\beta}^{\prime}$. It is easy to see that this twisting process does not change the 1-semisimplicity of the $\mathbb{Z}$-action on $G$, so the kernel of $H^{1}\left(\mathbb{Z}, T_{\beta}^{\prime}\right) \rightarrow H^{1}\left(\mathbb{Z}, G_{i \circ \beta}\right)$ is finite. Note that $H^{1}\left(\mathbb{Z}, T_{\beta}\right) \rightarrow H^{1}\left(\mathbb{Z}, T_{\beta}^{\prime}\right)$ is injective, hence the kernel of $H^{1}\left(\mathbb{Z}, T_{\beta}\right) \rightarrow H^{1}\left(\mathbb{Z}, G_{i \circ \beta}\right)$ is finite. This proves that $i_{1}^{-1}([\alpha])$ is finite.

"(iv) $\Rightarrow$ (iii)". Obvious.

"(iii) $\Rightarrow$ (i)". Suppose that the kernel of $H^{1}(\mathbb{Z}, T) \rightarrow H^{1}(\mathbb{Z}, G)$ is finite. By the commutative diagram (4.2), we know the kernel of $H^{1}\left(\mathbb{Z}, T^{\prime}\right) \rightarrow H^{1}\left(\mathbb{Z}, G^{\prime}\right)$ is finite. Also, by the commutative diagram (4.1), we know the kernel of $H^{1}\left(\mathbb{Z}, T_{t}\right) \rightarrow$ $H^{1}\left(\mathbb{Z}, G_{t}\right)$ is also finite. By Lemma 4.5 , the $\mathbb{Z}$-action on $G_{t}$ is 1 -semisimple. Hence the $\mathbb{Z}$-action on $G$ is 1 -semisimple.

Theorem 4.1 has the following corollaries.

Corollary 4.1. Let $G$ be a connected compact semisimple Lie group with a $\mathbb{Z}$ module structure, and let $T$ be a maximal torus of $G_{0}^{\mathbb{Z}}$. Then the canonical map $i_{1}: H^{1}(\mathbb{Z}, T) \rightarrow H^{1}(\mathbb{Z}, G)$ is surjective with finite fibers.

Proof. Since $G$ is compact semisimple, the automorphism group $\operatorname{Aut}(G)$ of $G$ is compact. So the action of each $\sigma \in \operatorname{Aut}(G)$ on $G$ is semisimple. As a sub-action of $\operatorname{Aut}(G)$, the $\mathbb{Z}$-action on $G$ is semisimple, hence 1-semisimple, and the corollary follows from Theorem 4.1 immediately.

Corollary 4.2. Let $G$ be a connected compact Lie group with a $\mathbb{Z}$-module structure, and let $T$ be a maximal torus of $G_{0}^{\mathbb{Z}}$. Suppose the $\mathbb{Z}$-action on $G$ is 1-semisimple. Then every connected component of $H^{0}(\mathbb{Z}, G / T)$ is a closed homogeneous submanifold of $G / T$, and the restriction of the coboundary operator $\delta: H^{0}(\mathbb{Z}, G / T) \rightarrow$ $H^{1}(\mathbb{Z}, T)$ to each connected component of $H^{0}(\mathbb{Z}, G / T)$ is constant.

Proof. From the exact sequence

$$
H^{0}(\mathbb{Z}, G / T) \stackrel{\delta}{\rightarrow} H^{1}(\mathbb{Z}, T) \stackrel{i_{1}}{\rightarrow} H^{1}(\mathbb{Z}, G)
$$

we know that the image of $\delta$ coincides with the kernel of $i_{1}$, which is finite by Theorem 4.1. Therefore the image of each connected component of $H^{0}(\mathbb{Z}, G / T)$ is finite. But under the identification $H^{1}(\mathbb{Z}, T)$ with $T, \delta$ is continuous. This forces that the restriction of $\delta$ to each connected component of $H^{0}(\mathbb{Z}, G / T)$ is constant. 
Let $M$ be a connected component of $H^{0}(\mathbb{Z}, G / T)$, and suppose that $\delta(M)=$ $\{t\} \subset T \cong H^{1}(\mathbb{Z}, T)$. We compute the subset $\delta^{-1}(t)$ of $G / T$. Choose a point $g_{0} T \in$ $M$. Since $\delta: H^{0}(\mathbb{Z}, G / T) \rightarrow H^{1}(\mathbb{Z}, T) \cong T$ has the expression $\delta(g T)=g^{-1} \sigma(g)$, we have $g_{0}^{-1} \sigma\left(g_{0}\right)=t$. Now suppose $g T \in H^{0}(\mathbb{Z}, G / T)$; then

$$
\begin{aligned}
& \delta(g T)=g^{-1} \sigma(g)=t \\
\Leftrightarrow & \left(g_{0}^{-1} g\right)^{-1} g_{0}^{-1} \sigma\left(g_{0}\right) \sigma\left(g_{0}^{-1} g\right)=t \\
\Leftrightarrow & t \sigma\left(g_{0}^{-1} g\right) t^{-1}=g_{0}^{-1} g .
\end{aligned}
$$

Since $H=\left\{h \in G \mid t \sigma(h) t^{-1}=h\right\}$ is a closed subgroup of $G$ containing $T, \delta^{-1}(t)$ is diffeomorphic to $H / T$. Hence $M$, as a connected component of $\delta^{-1}(t)$, is diffeomorphic to $H_{0} / T$, which is a closed homogeneous submanifold of $G / T$. This proves the corollary.

\section{Nonabelian COHOMOLOGY OF $\mathbb{Z} / n \mathbb{Z}$ WITH COEFFICIENTS IN LIE GROUPS}

Let $A=\mathbb{Z} / n \mathbb{Z}, G$ be a connected Lie group with an $A$-module structure. Then all assertions which have been proved in Section 3 hold for $H^{1}(A, G)$. In this section we prove further properties of $H^{1}(\mathbb{Z} / n \mathbb{Z}, G)$. We first introduce a notion.

Let $H$ be a connected Lie group. A subgroup $T$ of $H$ is a maximal compact torus of $H$ if $T$ is a compact torus and there is no other compact torus $T^{\prime}$ of $H$ such that $T \varsubsetneqq T^{\prime}$. Note that if $H$ is compact, this notion coincides with the commonly used notion of maximal torus.

It is obvious that maximal compact tori always exist. We claim that they are unique up to conjugation.

Proposition 5.1. Let $H$ be a connected Lie group, $T$ a maximal compact torus of $H$. Then for any compact torus $T^{\prime}$ of $H$, there exists an $h \in H$ such that $h T^{\prime} h^{-1} \subset T$. In particular, any two maximal compact tori of $H$ are conjugate.

Proof. Let $K$ be a maximal compact subgroup of $G$ containing $T$. By Fact 3.1, there is an $h_{1} \in H$ such that $h_{1} T^{\prime} h_{1}^{-1} \subset K$. Since $K$ is connected compact and $T$ is a maximal torus of $K$, there is a $k \in K$ such that $\left(k h_{1}\right) T^{\prime}\left(k h_{1}\right)^{-1} \subset T$.

Theorem 5.1. Let $G$ be a connected Lie group with a $\mathbb{Z} / n \mathbb{Z}$-module structure, and let $T$ be a maximal compact torus of $G_{0}^{\mathbb{Z} / n \mathbb{Z}}$. Then the canonical map $i_{1}$ : $H^{1}(\mathbb{Z} / n \mathbb{Z}, T) \rightarrow H^{1}(\mathbb{Z} / n \mathbb{Z}, G)$ is surjective.

Proof. Let $\sigma$ be a generator of $\mathbb{Z} / n \mathbb{Z}$. Then under the identification $Z^{1}(\mathbb{Z} / n \mathbb{Z}, G) \cong$ $Z=\left\{g \in G \mid g \sigma(g) \cdots \sigma^{n-1}(g)=e\right\}$ (see Section 2), two cocycles $z, z^{\prime} \in Z$ are cohomologous if there exists $g \in G$ such that $z^{\prime}=g^{-1} z \sigma(g)$. So to prove the theorem, it is sufficient to show that for each $z \in Z$, there exists $g \in G$ such that $g^{-1} z \sigma(g) \in T$.

Let $z \in Z$. By Theorem 3.1, there exists a maximal compact subgroup $K$ of $G$ which is also a $\mathbb{Z} / n \mathbb{Z}$-submodule of $G$, and there also exists $g_{1} \in G$ such that $g_{1}^{-1} z \sigma\left(g_{1}\right) \in K$.

Denote $z_{1}=g_{1}^{-1} z \sigma\left(g_{1}\right)$. Let $T^{\prime}$ be a maximal compact torus of $K_{0}^{\mathbb{Z} / n \mathbb{Z}}$. Since $\sigma$ is of finite order, it is semisimple, hence 1-semisimple. By Theorem 4.1, there is a $k \in K$ such that $k^{-1} z_{1} \sigma(k) \in T^{\prime}$. 
Denote $z_{2}=k^{-1} z_{1} \sigma(k)$. Since $T^{\prime}$ is a compact torus of $G_{0}^{\mathbb{Z} / n \mathbb{Z}}$, by Proposition 5.1 , there exists a $g_{2} \in G_{0}^{\mathbb{Z} / n \mathbb{Z}}$ such that $g_{2}^{-1} T^{\prime} g_{2} \subset T$. In particular, $g_{2}^{-1} z_{2} g_{2} \in T$. Denote $g=g_{1} k g_{2}$, and notice that $\sigma\left(g_{2}\right)=g_{2}$; we have $g^{-1} z \sigma(g) \in T$. This proves the theorem.

Corollary 5.1. Let $G$ be a connected and simply connected solvable Lie group with $a \mathbb{Z} / n \mathbb{Z}$-module structure. Then $H^{1}(\mathbb{Z} / n \mathbb{Z}, G)$ is trivial.

Proof. Let $T$ be a maximal compact torus of $G_{0}^{\mathbb{Z} / n \mathbb{Z}}$. By Theorem 5.1, it is sufficient to show that $H^{1}(\mathbb{Z} / n \mathbb{Z}, T)$ is trivial. But a connected and simply connected solvable Lie group has no nontrivial compact subgroup ([6], Chapter XII, Theorem 2.3). So $T$ is trivial, and hence $H^{1}(\mathbb{Z} / n \mathbb{Z}, T)$ is trivial.

Theorem 5.2. Let $G$ be a connected Lie group with a $\mathbb{Z} / n \mathbb{Z}$-module structure, and let $\sigma$ be a generator of $\mathbb{Z} / n \mathbb{Z}$. Let $Z=\left\{g \in G \mid g \sigma(g) \cdots \sigma^{n-1}(g)=e\right\}$. Then we have

(i) $Z$ has finitely many connected components, each of which is a closed submanifold of $G$;

(ii) Under the identification $Z^{1}(\mathbb{Z} / n \mathbb{Z}, G) \cong Z$, each cohomology class is a connected component of $Z$, that is, $H^{1}(\mathbb{Z} / n \mathbb{Z}, G)$ coincides with the set of all connected components of $Z$.

Theorem 5.2 is equivalent to Theorem 3.2 for the case that $A=\mathbb{Z} / n \mathbb{Z}$. In fact, the map $\rho: G \rightarrow G^{n}$ defined by $\rho(g)=\left(e, g, g \sigma(g), \cdots, g \sigma(g) \cdots \sigma^{n-2}(g)\right)$ is an embedding, and $\rho(Z)=Z^{1}(\mathbb{Z} / n \mathbb{Z}, G)$ under the natural identification $G^{n} \cong$ $\operatorname{Map}(\mathbb{Z} / n \mathbb{Z}, G)$. But we would like to provide another proof of Theorem 5.2 , without the use of Weil's Lemma.

Proof of Theorem 5.2. First we note that the formula

$$
\operatorname{Ad}\left(g \sigma(g) \cdots \sigma^{k-1}(g)\right) d \sigma^{k}=(\operatorname{Ad}(g) d \sigma)^{k}
$$

holds for every $g \in G$ and every positive integer $k$. In fact, with respect to the $\mathbb{Z}$-module structure on $G$ defined by $(m, h) \mapsto \sigma^{m}(h)$, there is a cocycle $\alpha: \mathbb{Z} \rightarrow G$ such that $\alpha(m)=g \sigma(g) \cdots \sigma^{m-1}(g)$ for $m>0$. Twisting the $\mathbb{Z}$-module $G$ using the cocycle $\alpha$, we know that $(m, h) \mapsto \alpha(m) \sigma^{m}(h) \alpha(m)^{-1}$ is an action of $\mathbb{Z}$ on $G$. Differentiating both sides of the equation $\alpha(k) \sigma^{k}(h) \alpha(k)^{-1}=\left(g \sigma(h) g^{-1}\right)^{k}$, we get the desired formula.

Now let $z \in Z$. Denote $\mathfrak{a}=\operatorname{ker}(\operatorname{Ad}(z) d \sigma-1)$ and $\mathfrak{b}=\operatorname{Im}(\operatorname{Ad}(z) d \sigma-1)$. By the above formula, we have $(\operatorname{Ad}(z) d \sigma)^{n}=1$. So $\operatorname{Ad}(z) d \sigma$ is semisimple, and then $\mathfrak{g}=\mathfrak{a} \oplus \mathfrak{b}$. We construct a smooth map $F_{z}: \mathfrak{a} \oplus \mathfrak{b} \rightarrow G$ by

$$
F_{z}(X, Y)=e^{-Y} e^{X} z \sigma\left(e^{Y}\right) .
$$

Then it is easy to compute the differential $d F_{z}(0,0): \mathfrak{a} \oplus \mathfrak{b} \rightarrow T_{z} G$ as

$$
d F_{z}(0,0)(X, Y)=\left(d r_{z}\right)_{e}(X+(\operatorname{Ad}(z) d \sigma-1)(Y)),
$$

where $r_{z}$ is the right translation on $G$ induced by $z$. Since $\operatorname{Ad}(z) d \sigma$ is semisimple, the restriction of $\operatorname{Ad}(z) d \sigma-1$ on $\mathfrak{b}=\operatorname{Im}(\operatorname{Ad}(z) d \sigma-1)$ is a linear automorphism. Hence $d F_{z}(0,0)$ is a linear isomorphism, and then $F_{z}$ is a local diffeomorphism at 
$(0,0)$. Choose an open neighborhood $U_{1}$ of $0 \in \mathfrak{a}$ and an open neighborhood $U_{2}$ of $0 \in \mathfrak{b}$ such that the restriction of $F_{z}$ to $U_{1} \times U_{2} \subset \mathfrak{a} \oplus \mathfrak{b}$ is a diffeomorphism onto an open neighborhood $V=F_{z}\left(U_{1} \times U_{2}\right)$ of $z \in G$. Shrinking $U_{1}$ if necessary, we may assume that $X \in U_{1}, e^{n X}=e$ implies $X=0$. For $X \in U_{1}, Y \in U_{2}$, we have

$$
\begin{aligned}
& F_{z}(X, Y) \sigma\left(F_{z}(X, Y)\right) \cdots \sigma^{n-1}\left(F_{z}(X, Y)\right) \\
= & e^{-Y} e^{X} z \sigma\left(e^{X} z\right) \cdots \sigma^{n-1}\left(e^{X} z\right) e^{Y} \\
= & e^{-Y} e^{X} e^{\operatorname{Ad}(z) d \sigma(X)} e^{\operatorname{Ad}(z \sigma(z)) d \sigma^{2}(X)} \cdots e^{\operatorname{Ad}\left(z \sigma(z) \cdots \sigma^{n-2}(z)\right) d \sigma^{n-1}(X)} e^{Y} \\
= & e^{-Y} e^{X} e^{\operatorname{Ad}(z) d \sigma(X)} e^{(\operatorname{Ad}(z) d \sigma)^{2}(X)} \cdots e^{(\operatorname{Ad}(z) d \sigma)^{n-1}(X)} e^{Y} \\
= & e^{-Y} e^{n X} e^{Y} .
\end{aligned}
$$

So $F_{z}(X, Y) \in Z \Leftrightarrow X=0$, that is,

$$
Z \cap V=F_{z}\left(\{0\} \times U_{2}\right)=\left\{e^{-Y} z \sigma\left(e^{Y}\right) \mid Y \in U_{2}\right\} .
$$

This shows that each connected component of $Z$ is a submanifold of $G$, which is necessarily closed by the definition of $Z$, and that each cohomology class is open in $Z$. So each cohomology class is also closed in $Z$. But the fact that $G$ is connected implies that cohomology classes are connected. Hence each cohomology class is in fact a connected component of $Z$. This proves the theorem.

Hence we get a new proof of the following assertion, which was first proved in [3] using real analytic geometry.

Corollary 5.2 ([3], Theorem 1.1). Let $G$ be a connected Lie group, and let $n$ be a positive integer. Then each connected component of the set $\left\{g \in G \mid g^{n}=e\right\}$ is a conjugacy class of $G$.

Proof. Consider the $\mathbb{Z} / n \mathbb{Z}$-module $G$ for which $\mathbb{Z} / n \mathbb{Z}$ acts trivially on $G$ and apply Theorem 5.2.

Remark 5.1. The idea of the construction of the map $F_{z}$ in the proof of Theorem 5.2 is due to Professor K.-H. Neeb.

We also have

Proposition 5.2. Let $G$ be a connected Lie group with a $\mathbb{Z} / n \mathbb{Z}$-module structure, and let $T$ be a maximal compact torus of $G_{0}^{\mathbb{Z} / n \mathbb{Z}}$. Then every connected component of $H^{0}(\mathbb{Z} / n \mathbb{Z}, G / T)$ is a closed homogeneous submanifold of $G / T$, and the restriction of the coboundary operator $\delta: H^{0}(\mathbb{Z} / n \mathbb{Z}, G / T) \rightarrow H^{1}(\mathbb{Z} / n \mathbb{Z}, T)$ to each connected component of $H^{0}(\mathbb{Z} / n \mathbb{Z}, G / T)$ is constant.

Proof. Similar to the proof of Corollary 4.2, using the fact that $H^{1}(\mathbb{Z} / n \mathbb{Z}, T)$ is finite.

\section{Twisted Conjugate actions of Lie groups}

Let $G$ be a connected Lie group, and let $\sigma$ be an automorphism of $G$. The twisted conjugate action of $G$ on itself associated with $\sigma$ is defined by $\tau_{g}(h)=g h \sigma(g)^{-1}$. An orbit of the twisted conjugate action is called a twisted orbit. If $\sigma$ is the identity, the associated twisted conjugate action is the adjoint action of $G$. 
The notion of twisted conjugate action was first introduced in [1, 2], where the authors considered the problem of embedding symmetric spaces into Lie groups. For a connected Lie group $G$ with an involution $\sigma$ such that $G^{\sigma}$ is compact, $G / G^{\sigma}$ has a structure of Riemannian symmetric space. It was proved in [1] that every connected component of the set $R=\left\{g \in G \mid \sigma(g)=g^{-1}\right\}$ is a closed submanifold of $G$. This is in fact a particular case of item (i) of Theorem 5.2 in this paper. It was also proved in [1] that the identity component of $R$ coincides with $P=\exp (\mathfrak{p})$, where $\mathfrak{p}$ is the eigenspace of $d \sigma$ in the Lie algebra $\mathfrak{g}$ of $G$ with eigenvalue -1 . This gave an isomorphism $G / G^{\sigma} \cong P$.

In this section we consider general properties of twisted conjugate actions of Lie groups. Letting $G$ be a Lie group acts smoothly on two manifolds $M_{1}$ and $M_{2}$ by $\rho_{i}: G \times M_{i} \rightarrow M_{i}(i=1,2)$. The two actions $\rho_{1}$ and $\rho_{2}$ are equivalent if there exists an equivariant diffeomorphism $\varphi: M_{1} \rightarrow M_{2}$, that is, $\varphi\left(\rho_{1}(g, x)\right)=\rho_{2}(g, \varphi(x))$ for every $g \in G$ and $x \in M_{1}$.

For a Lie group $G$, we denote the automorphism group and the inner automorphism group of $G$ by $\operatorname{Aut}(G)$ and $\operatorname{Inn}(G)$, respectively.

Proposition 6.1. Let $G$ be a connected Lie group. Then automorphisms of $G$ belonging to the same coset of $\operatorname{Inn}(G)$ in $\operatorname{Aut}(G)$ induce equivalent twisted conjugate actions.

Proof. Let $\sigma, \sigma^{\prime} \in \operatorname{Aut}(G)$, and denote the twisted conjugate actions associated with $\sigma$ and $\sigma^{\prime}$ be $\tau$ and $\tau^{\prime}$, respectively. Suppose there exists $g \in G$ such that $\sigma=\operatorname{Inn}(g) \circ \sigma^{\prime}$, where $\operatorname{Inn}(g)$ is the inner automorphism of $G$ induced by $g$. Then $r_{g} \circ \tau_{h}(k)=h k \sigma(h)^{-1} g=h k\left(g \sigma^{\prime}(h) g^{-1}\right)^{-1} g=h k g \sigma^{\prime}(h)^{-1}=\tau_{h}^{\prime} \circ r_{g}(k)$ for every $h, k \in G$. Hence the right multiplication $r_{g}: G \rightarrow G$ is equivariant. This proves the proposition.

Proposition 6.1 implies that twisted conjugate actions associated with inner automorphisms are equivalent to the adjoint action. In particular, if $G$ is semisimple, there are only finitely many equivalent types of actions appearing among twisted conjugate actions, due to the fact that $\operatorname{Aut}(G) / \operatorname{Inn}(G)$ is finite for $G$ semisimple. Even then, new types of actions do appear.

Proposition 6.2. Let $G$ be a connected Lie group with an automorphism $\sigma$. If $\sigma$ is not an inner automorphism, then the twisted conjugate action $\tau$ associated with $\sigma$ has no fixed point.

Proof. Suppose $\tau$ has a fixed point $h \in G$, that is, $\tau_{g}(h)=g h \sigma(g)^{-1}=h$ for every $g \in G$. Then $\sigma(g)=h^{-1} g h$ for every $g \in G$, a contradiction.

Since the identity element is a fixed point of the adjoint action, Proposition 6.2 implies that twisted conjugate actions associated with automorphisms which are not inner are not equivalent to the adjoint action.

Now we use some results about nonabelian cohomology of cyclic groups proved in previous sections to prove some properties on the structure of orbits of twisted conjugate actions.

Theorem 6.1. Let $G$ be a connected compact Lie group, and let $\sigma \in \operatorname{Aut}(G)$. Let $T$ be a maximal torus of $G_{0}^{\sigma}$. Then the following statements are equivalent:

(i) $\sigma$ is 1-semisimple;

(ii) $O \cap T$ is nonempty for every twisted orbit $O$ of $G$ associated with $\sigma$; 
(iii) $O_{e} \cap T$ is finite, where $O_{e}$ is the twisted orbit through the identity element $e$;

(iv) $O \cap T$ is finite for every twisted orbit $O$ of $G$ associated with $\sigma$.

Proof. Define the $\mathbb{Z}$-module structure on $G$ by $(m, g) \mapsto \sigma^{m}(g)$. Then by the relation between twisted conjugate actions and nonabelian cohomology (see Section 2 ), (ii) is equivalent to the fact that $H^{1}(\mathbb{Z}, T) \rightarrow H^{1}(\mathbb{Z}, G)$ is surjective, (iii) is equivalent to the fact that the kernel of $H^{1}(\mathbb{Z}, T) \rightarrow H^{1}(\mathbb{Z}, G)$ is finite, and (iv) is equivalent to the fact that all fibers of $H^{1}(\mathbb{Z}, T) \rightarrow H^{1}(\mathbb{Z}, G)$ are finite. Hence the theorem follows immediately from Theorem 4.1.

Theorem 6.2. Let $G$ be a connected Lie group, and let $\sigma$ be an automorphism of $G$ of finite order. Let $Z=\left\{g \in G \mid g \sigma(g) \cdots \sigma^{n-1}(g)=e\right\}$, where $n$ is a positive integer which is divisible by the order of $\sigma$. Then

(i) $Z$ has finitely many connected components, each of which is a twisted orbit;

(ii) For every maximal compact torus $T$ of $G_{0}^{\sigma}$ and every twisted orbit $O$ contained in $Z, O \cap T$ is nonempty and finite;

(iii) There exists a maximal compact subgroup $K$ of $G$ which is $\sigma$-invariant. For every such $K$ and every twisted orbit $O$ of $G$ contained in $Z, O \cap K$ is a twisted orbit of $K$.

Proof. Define the $\mathbb{Z} / n \mathbb{Z}$-module structure on $G$ by $(m, g) \mapsto \sigma^{m}(g)$. Then under the identification $Z^{1}(\mathbb{Z} / n \mathbb{Z}, G) \cong Z$ as explained in Section 2 , every cohomology class is a twisted orbit in $Z$. So (i) follows from Theorem 5.2, (ii) follows from Theorem 5.1 and the fact that $H^{1}(\mathbb{Z} / n \mathbb{Z}, T)$ is finite, and (iii) follows from Theorem 3.1.

\section{REFERENCES}

[1] An, J., Wang, Z., On the realization of Riemannian symmetric spaces in Lie groups, Topology Appl., 153 (2006), no. 7, 1008-1015. MR2203015 (2006k:53081)

[2] An, J., Wang, Z., On the realization of Riemannian symmetric spaces in Lie groups, II, Topology Appl., 153 (2006), no. 15, 2943-2947. MR2248396 (2007g:53055)

[3] An, J., Wang, Z., Curve Selection Lemma for semianalytic sets and conjugacy classes of finite order in Lie groups, preprint, math.GR/0506160.

[4] An, J., Wang, Z., Yan, K., A generalization of random matrix ensemble I: general theory, Pacific J. Math., to appear, math-ph/0502020.

[5] An, J., Wang, Z., Yan, K., A generalization of random matrix ensemble II: concrete examples and integration formulae, preprint, math-ph/0502021.

[6] Hochschild, G., The structrue of Lie groups, Holden-Day, San Francisco, 1965. MR0207883 $(34: 7696)$

[7] Kobayashi, S., Transformation groups in differential geometry, Springer-Verlag, New YorkHeidelberg, 1972. MR0355886 (50:8360)

[8] Kobayashi, S., Nomizu, K., Foundations of differential geometry, Vol. II, John Wiley \& Sons, Inc., New York-London-Sydney, 1969. MR0238225 (38:6501)

[9] Neukirch, J., Schmidt, A., Wingberg, K., Cohomology of number fields, Springer-Verlag, Berlin, 2000. MR1737196 (2000j:11168)

[10] Platonov, V., Rapinchuk, A., Algebraic groups and number theory, Academic Press, Boston, 1994. MR1278263 (95b:11039)

[11] Samelson, H., On curvature and characteristic of homogeneous spaces, Michigan Math. J., 5 (1958), 13-18. MR0103509 (21:2277)

[12] Serre, J.-P., Local fields, Springer-Verlag, New York-Berlin, 1979. MR554237 (82e:12016)

[13] Serre, J.-P., Galois cohomology, Springer-Verlag, Berlin, 1997. MR1466966 (98g:12007) 
[14] Serre, J.-P., Lie algebras and Lie groups, Springer-Verlag, Berlin, 1992. MR1176100 (93h:17001)

[15] Weil, A., Demonstration topologique d'un théorème fondamental de Cartan, C. R. Acad. Sci. Paris, 200 (1935), 518-520.

School of Mathematical Science, Peking University, Beijing, 100871, People's RePUBLIC OF ChINA

E-mail address: anjinpeng@gmail.com

Current address: Department of Pure Mathematics, University of Waterloo, Waterloo, Ontario, Canada N2L 3G1

School of Mathematical Science, Peking University, Beijing, 100871, People's RePUBLIC OF CHINA

E-mail address: zdwang@pku.edu.cn 